\title{
Diffusion Kinetics in Radiation Chemistry. II. One-Radical-One-Solute Model; Calculations
}

\author{
Aron Kuppermann aNd Geneva G. Betrord \\ Noyes Chemical Laboratory, University of Illinois, Urbana, Illinois*
}

(Received August 29, 1961)

\begin{abstract}
The general diffusion-kinetic equations are applied to a one-radical-one-solute model of the radiolysis of dilute aqueous solutions. The validity of the prescribed diffusion approximation is examined. Results of calculations of the effect on the molecular and radical yields of the following parameters are given: solute concentration, solute depletion, shape of initial radical distribution, radical density, diffusion coefficients, and rate constants. Conditions under which a straight track of equal and equidistant spherical spurs can be replaced by either isolated spherical spurs or an axially homogeneous cylindrical track are examined.
\end{abstract}

\section{INTRODUCTION}

$\mathbf{I}^{\mathrm{N}}$ $\mathrm{N}$ the preceding paper, which henceforth will be referred to as paper I, a generalized formulation of the diffusion-kinetic model in radiation chemistry was given. Before we attempt to apply this formulation to a realistic mechanism, such as that given in Sec. 2.8 of paper I, it is convenient to gain insight into the quantitative properties of the diffusion-kinetic equations by considering a simplified mechanism. One such mechanism is the following. Assume that passage of the highenergy radiation produces, by the beginning of the chemical stage (see paper I), a single kind of radical $R$ and that there is only one solute $S$ dissolved in the medium. The radicals are capable of reacting either with each other to give molecules $R_{2}$ (which are assumed to be distinguishable from the molecules of the medium) or with the solute to give product RS. The reaction equations are

$$
\begin{array}{ll}
\mathrm{R}+\mathrm{R} \rightarrow \mathrm{R}_{2} & k_{\mathrm{RR},} \\
\mathrm{R}+\mathrm{S} \rightarrow \mathrm{RS} & k_{\mathrm{RS} .}
\end{array}
$$

Such a model has been considered previously. ${ }^{1-3}$ Samuel and Magee $^{1}$ treated the model analytically, using the prescribed diffusion approximation and neglecting the presence of solute. In this paper we present the results of numerical calculations on this model. A brief summary of these results has been published elsewhere. ${ }^{3}$ The diffusion-kinetic equations are given in Sec. 2.1. The calculations are used to describe the space-time history of a spherically symmetric spur and an axially homogeneous cylindrically symmetric track (Sec. 2.2) and to test the validity of the prescribed diffusion approximation (Sec. 3). They are also used

\footnotetext{
* Work partially supported by the U. S. Atomic Energy Commission. Presented in part at the 136th Meeting of the American Chemical Society in Atlantic City, September, 1959.

1 A. H. Samuel and J. L. Magee, J. Chem. Phys. 21, 1080 (1953).

2 See references $7-9$ and $20-23$ of paper $I$.

"For a summary of past work, see A. Kuppermann, "Diffusion kinetics in radiation chemistry," in Actions Chimiques et Biologiques des Radiations, edited by M. Haissinsky (Masson et Cie., Paris, 1961), pp. 85-166.
}

to investigate the effect on yields of solute concentration and depletion (Sec. 4), shape of initial radical distribution (Sec. 5), and radical density, diffusion coefficient and rate constants (Sec. 6).

Conditions under which a straight track of equal and equidistant spherical spurs can be replaced by either isolated spherical spurs or an axially homogeneous cylindrical track are examined in Sec. 6.3. A discussion of the comparison of these calculations with experiment is left to a subsequent paper in this series.

\section{SOLUTION OF DIFFUSION-KINETIC EQUATIONS}

\subsection{Diffusion-Kinetic and Yield Equations}

Let us consider the situation in which the dose-rate (rate of energy absorption by the medium) is so low that the chemical effects of the high-energy radiation can be pictured as a sum of the separate effects of individual quanta or particles (Sec. 2.4, paper I). Let $c_{\mathrm{R}}$ and $c_{\mathrm{S}}$ represent the generalized concentrations (probability densities; cf. Sec. 2.2, paper I) of $R$ and $S$ at point $P$ and time $t$ after the onset of the chemical stage. Then the diffusion-kinetic equations representing the space-time behavior of the track of an individual quantum or particle are

$$
\left\{\begin{array}{l}
\partial c_{\mathrm{R}} / \partial t=D_{\mathrm{R}} \nabla^{2} c_{\mathrm{R}}-k_{\mathrm{RR}} c_{\mathrm{R}}{ }^{2}-k_{\mathrm{RS}} c_{\mathrm{R}} c_{\mathrm{S}}, \\
\partial c_{\mathrm{S}} / \partial t=D_{\mathrm{s}} \nabla^{2} c_{\mathrm{S}}-k_{\mathrm{RS}} c_{\mathrm{R}} c_{\mathrm{s}} .
\end{array}\right.
$$

The quantities $D_{\mathbb{R}}$ and $D_{\mathrm{S}}$ are the diffusion coefficients of $\mathrm{R}$ and $\mathrm{S}$, respectively; $k_{\mathrm{RR}}$ and $k_{\mathrm{RS}}$ are the secondorder rate constants for the disappearance of radicals $R$ by reactions [1] and [2], respectively (they are assumed time independent in this paper); $\nabla^{2}$ is the usual Laplacian operator.

The problem of initial distributions and boundary conditions has been examined in detail in Sec. 2.5 of paper I. In this paper we will consider the extreme cases of isolated spherically symmetric spurs and axially homogeneous cylindrically symmetric tracks as approximate models for describing the chemical effects of radiations of low and high LET, respectively. In both 


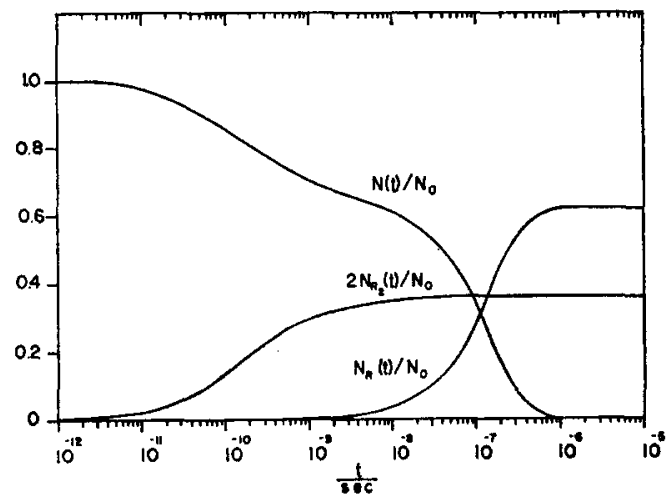

Fig. 1. Variation of $N(t) / N_{0}, 2 N_{\mathrm{R}_{2}}(t) / N_{0}$, and $N_{\mathrm{R}}(t) / N_{0}$ with time for a spherical spur. Gaussian initial distribution. $N_{0}=12$ radic, $r_{0}=10 \mathrm{~A}, D_{\mathrm{R}}=4 \times 10^{-5} \mathrm{~cm}^{2} / \mathrm{sec}, D_{\mathrm{g}}=4 \times 10^{-6} \mathrm{~cm}^{2} /$ sec, $k_{\mathrm{RR}}=k_{\mathrm{Rs}}=10^{-11} \mathrm{~cm}^{8} /(\mathrm{sec} \times$ radic $), c_{\mathrm{B} 0}=10^{-8} \mathrm{~mole} / \mathrm{l}$.

these cases, a single space coordinate is sufficient to describe the problem; it is the distance $r$ to the center of the spherically symmetrical spur or to the axis of the cylindrically symmetrical track (Sec. 4.2, paper I). The Laplacian operator for these cases is given by

$$
\nabla^{2}=\partial^{2} / \partial r^{2}+(\alpha / r)(\partial / \partial r) ; \alpha=\left\{\begin{array}{l}
2 \text { for spherical case } \\
1 \text { for cylindrical case }
\end{array}\right.
$$

The initial distribution for the solute is

$$
c_{\mathrm{S}}(r, 0)=c_{\mathrm{S}_{0}}
$$

i.e., initially the solute is homogeneously distributed with concentration $c_{\mathbb{B}_{0}}$. The initial distribution for the radicals is assumed to be either Gaussian or rectangular, as described by Eqs. (5) and (6), respectively.

$$
\begin{gathered}
c_{\mathrm{R}}(r, 0)=c_{\mathrm{R}}(0,0) \exp \left(-r^{2} / 2 r_{0}^{2}\right) . \\
c_{\mathrm{R}}(r, 0)=\left\{\begin{array}{cc}
c_{\mathrm{R}}(0,0) & \text { for } r<r_{0} ; \\
0 & \text { for } r>r_{0} .
\end{array}\right.
\end{gathered}
$$

For either initial distribution, $r_{0}$ is called the initial radius of the spur or track.

For both the spherical and cylindrical cases the medium is assumed to be infinite, as justified in Sec. 2.5 of paper $I$. The boundary conditions are then given by

$$
\begin{aligned}
& c_{\mathrm{B}}(\infty, t)=c_{\mathrm{B}_{0}} ; \quad \partial c_{\mathrm{S}}(0, t) / \partial r=0 ; \\
& c_{\mathrm{R}}(\infty, t)=0 ; \quad \partial c_{\mathrm{R}}(0, t) / \partial r=0 .
\end{aligned}
$$

It is useful, at this stage, to give some relationships between the several initial distribution parameters. The initial root mean square radius $r_{0}(0)$ is given by Eqs. (9) and (10) for the Gaussian and rectangular cases, respectively.

$$
\begin{aligned}
& r_{\sigma}(0)=(1+\alpha)^{t} r_{0}, \\
& r_{\sigma}(0)=[(1+\alpha) /(3+\alpha)]^{1} r_{0} .
\end{aligned}
$$

The parameter $\alpha$ is the same as in Eq. (3). For the Gaussian distribution, the initial half-radius $r_{\frac{1}{3}}(0)$ [the value of $r$ for which $\left.c_{R}(r, 0)=\frac{1}{2} c_{R}(0,0)\right]$ is given by

$$
r_{\frac{1}{3}}(0)=(2 \ln 2)^{\frac{1}{3}} r_{0} \simeq 1.18 r_{0} .
$$

Let $N_{0}$ be the initial number of radicals in a spur for the spherical case and the initial linear radical density (number of radicals per unit length of track) for the cylindrical one. The initial radical concentration $c_{R}(0,0)$ at the center of the spur or axis of the track can be obtained from

$$
c_{\mathrm{R}}(0,0)=N_{0} / f(\alpha, \beta) r_{0}^{\alpha+1},
$$

where $f(\alpha, \beta)$ is defined by

$$
\begin{gathered}
f(\alpha, \beta)=\frac{1-(-1)^{\beta}}{2}(2 \pi)^{(\alpha+1) / 2}+\frac{1+(-1)^{\beta}}{2} \frac{2^{\alpha} \pi}{\alpha+1} . \\
\beta=\left\{\begin{array}{l}
1 \text { for Gaussian initial distribution; } \\
0 \text { for rectangular initial distribution. }
\end{array}\right.
\end{gathered}
$$

For the spherical case, let $N(t), N_{\mathrm{R}_{2}}(t)$ and $N_{\mathrm{R}}(t)$ be respectively the number of radicals at time $t$ and the number of $R_{2}$ and RS molecules formed up to time $t$. For the cylindrical case, let these symbols represent the equivalent linear densities; i.e., the numbers of radicals or molecules per unit length of track. Then

$$
N(t)=\int_{0}^{\infty} c_{\mathrm{R}}(r, t) 2^{\alpha} \pi r^{\alpha} d r
$$

$$
\begin{gathered}
N_{\mathrm{R}_{2}}(t)=\frac{1}{2} \int_{0}^{t} d \theta \int_{0}^{\infty} k_{\mathrm{RR}} C_{\mathrm{R}}{ }^{2}(r, \theta) 2^{\alpha} \pi r^{\alpha} d r, \\
N_{\mathrm{R}}(t)=\int_{0}^{t} d \theta \int_{0}^{\infty} k_{\mathrm{RS}} c_{\mathrm{R}}(r, \theta) c_{\mathrm{S}}(r, \theta) 2^{\alpha} \pi r^{\alpha} d r, \\
N(t)+2 N_{\mathrm{R}_{2}}(t)+N_{\mathrm{R}}(t)=N(0)=N_{0} .
\end{gathered}
$$



Fig. 2. Variation of $N(t) / N_{0}, 2 N_{R_{0}}(t) / N_{0}$, and $N_{\mathbf{R}}(t) / N_{0}$ with time for a spherical spur. Gaussian initial distribution $c_{\mathrm{B}_{0}}=10^{-1}$ mole/1, other parameters the same as for Fig. 1. 
The quantities $N_{\mathrm{R}_{2}}(\infty)$ and $N_{\mathrm{R}}(\infty)$, which we will represent by $N_{\mathrm{R}_{2}}$ and $N_{\mathrm{R}}$, are related to the molecular and free radical yields, and can be calculated once $c_{\mathrm{R}}(r, t)$ and $c_{\mathrm{S}}(r, t)$ have been obtained from an integration of the diffusion-kinetic equations.

As described in Sec. 4.1 of paper I, it is convenient, for the purpose of performing numerical integrations, to introduce dimensionless quantities. In the calculations described in this paper, $D^{*}, r^{*}$, and $c^{*}$ were chosen as, respectively, $D_{\mathrm{R}}, a r_{0}$ and $b c_{\mathrm{R}}(0,0), a$ and $b$ having been given integer values between 1 and 10 inclusive for computational convenience. The dimensionless variables are then given by

$$
\begin{aligned}
r^{\prime} & =r / a r_{0}, \\
t^{\prime} & =t /\left(a^{2} r_{0}^{2} / D_{\mathrm{R}}\right), \\
c_{\mathrm{R}}{ }^{\prime}\left(r^{\prime}, t^{\prime}\right) & =\frac{c_{\mathrm{R}}\left[a r_{0} r^{\prime},\left(a^{2} r_{0} / D_{\mathrm{R}}\right) t^{\prime}\right]}{b c_{\mathrm{R}}(0,0)}, \\
c_{\mathrm{S}}\left(r^{\prime}, t^{\prime}\right) & =\frac{c_{\mathrm{S}}\left[a r_{0} r^{\prime},\left(a^{2} r_{0}{ }^{2} / D_{\mathrm{R}}\right) t^{\prime}\right]}{b c_{\mathrm{R}}(0,0)} .
\end{aligned}
$$

In terms of these variables, Eqs. (1) and (2) become

$$
\left\{\begin{array}{l}
\partial c_{\mathrm{R}}{ }^{\prime} / \partial t^{\prime}=\nabla^{\prime 2} c_{\mathrm{R}}{ }^{\prime}-k_{\mathrm{RR}}{ }^{\prime} c_{\mathrm{R}}{ }^{2}-k_{\mathrm{RS}}{ }^{\prime} c_{\mathrm{R}}{ }^{\prime} c_{\mathrm{S}}{ }^{\prime}, \\
\partial c_{\mathrm{S}}{ }^{\prime} / \partial t^{\prime}=D_{\mathrm{S}}{ }^{\prime} \nabla^{\prime 2} c_{\mathrm{S}}{ }^{\prime}-k_{\mathrm{RS}}{ }^{\prime} c_{\mathrm{R}}{ }^{\prime} c_{\mathrm{s}}{ }^{\prime},
\end{array}\right.
$$

where

$$
\nabla^{\prime 2}=\left(\partial^{2} / \partial r^{\prime 2}\right)+\left(\alpha / r^{\prime}\right)\left(\partial / \partial r^{\prime}\right) .
$$

The $k^{\prime}$ and $D^{\prime}$ are dimensionless rate constants and diffusion coefficients given by

$$
\begin{aligned}
& k_{\mathrm{RR}}{ }^{\prime}=a^{2} b k_{\mathrm{RR}} * ; \quad k_{\mathrm{RR}}^{*}=\frac{k_{\mathrm{RR}}}{D_{\mathrm{R}} /\left[r_{0}^{2} c_{\mathrm{R}}(0,0)\right]} \\
& k_{\mathrm{RS}}{ }^{\prime}=a^{2} b k_{\mathrm{RS}} * ; \quad k_{\mathrm{RS}}{ }^{*}=\frac{k_{\mathrm{RS}}}{D_{\mathrm{R}} /\left[r_{0}^{2} c_{\mathrm{R}}(0,0)\right]} \\
& D_{\mathrm{S}}{ }^{\prime}=D_{\mathrm{S}}^{*}=D_{\mathrm{S}} / D_{\mathrm{R}} .
\end{aligned}
$$

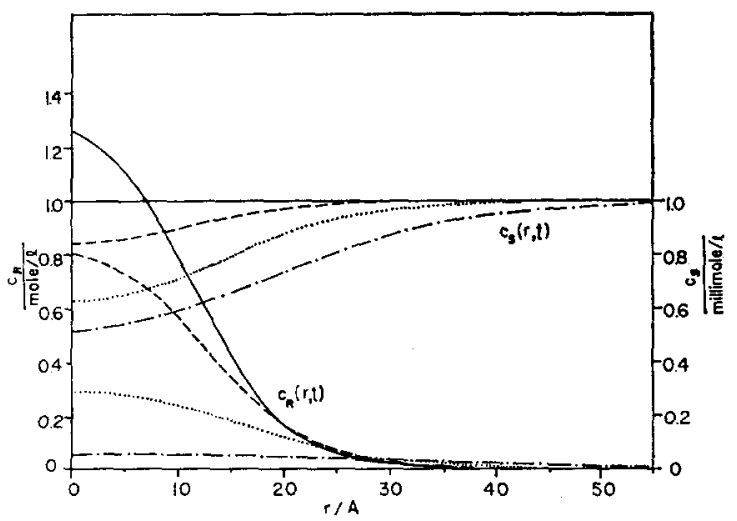

FIG. 3. Variation of $c_{\mathrm{R}}(r, t)$ and $c_{\mathrm{B}}(r, t)$ with $r$ for a spherical spur. Gaussian initial distribution. Same parameters as for Fig. 1. $\underline{-} t=0, N(t) / N_{0}=1 ;-t=2.8 \times 10^{-11} \mathrm{sec}, N(t) / N_{0}=0.94$ $\ldots t=1.4 \times 10^{-10} \mathrm{sec}, N(t) / N_{0}=0.83 ; \ldots . . . t=5.9 \times 10^{-10} \mathrm{sec}$ $N(t) / N_{0}=0.73$.

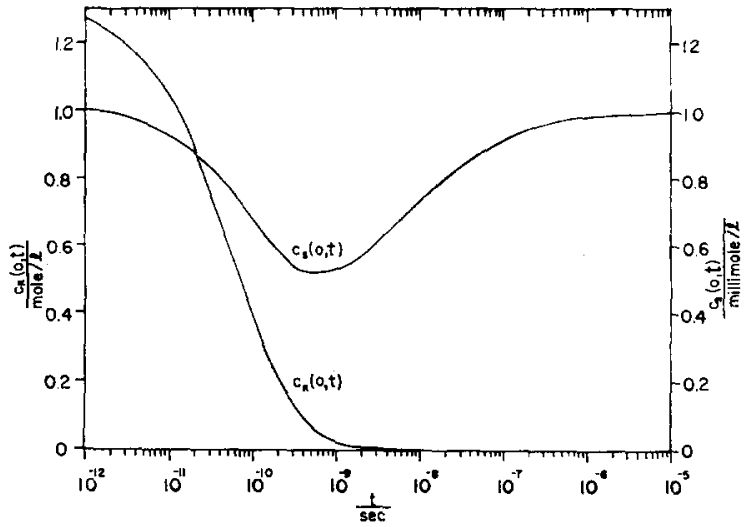

FIG. 4. Variation of $c_{\mathrm{R}}(0, t)$ and $c_{\mathrm{B}}(0, t)$ with time for a spherical spur. Gaussian initial distribution. Same parameters as in Fig. 1.

The initial distributions of Eqs. (4), (5), and (6) become

$$
\begin{aligned}
c_{\mathrm{S}}\left(r^{\prime}, 0\right) & =(1 / b) c_{\mathrm{S}_{0}}{ }^{*}, \text { where } c_{\mathrm{S}_{0}}{ }^{*}=c_{\mathrm{S}_{0}} / c_{\mathrm{R}}(0,0) ; \\
c_{\mathrm{R}}{ }^{\prime}\left(r^{\prime}, 0\right) & =\exp \left(-a^{2} r^{\prime 2} / 2\right) ; \\
c_{\mathrm{R}}{ }^{\prime}\left(r^{\prime}, 0\right) & =\left\{\begin{array}{l}
1 \text { for } r^{\prime}<1 / a, \\
0 \text { for } r^{\prime}>1 / a .
\end{array}\right.
\end{aligned}
$$

The boundary conditions transform into

$$
\begin{array}{ll}
c_{\mathrm{S}}{ }^{\prime}\left(\infty, t^{\prime}\right)=c_{\mathrm{S}_{0}} / a c_{\mathrm{R}}(0,0) ; & \partial c_{\mathrm{S}}{ }^{\prime}\left(0, t^{\prime}\right) / \partial r^{\prime}=0 ; \\
c_{\mathrm{R}}{ }^{\prime}\left(\infty, t^{\prime}\right)=0 ; & \partial c_{\mathrm{R}}{ }^{\prime}\left(0, t^{\prime}\right) / \partial r^{\prime}=0 .
\end{array}
$$

Equations (14) through (16) become

$$
\begin{aligned}
N(t) / N_{0}= & {\left[a^{\alpha+1} b / f(\alpha, \beta)\right] \int_{0}^{\infty} c_{\mathbf{R}^{\prime}}\left(r^{\prime}, t^{\prime}\right) 2^{\alpha} \pi r^{\prime \alpha} d r^{\prime}, } \\
2 N_{\mathbf{R}_{\mathbf{q}}}(t) / N_{0}= & {\left[a^{\alpha+1} b / f(\alpha, \beta)\right] \int_{0}^{t^{\prime}} d \theta^{\prime} } \\
\times \int_{0}^{\infty} k_{\mathrm{RR}}{ }^{\prime} c_{\mathrm{R}}{ }^{2}\left(r^{\prime}, \theta^{\prime}\right) 2^{\alpha} \pi r^{\prime \alpha} d r^{\prime} & \\
N_{\mathbf{R}}(t) / N_{0}= & {\left[a^{\alpha+1} b / f(\alpha, \beta)\right] \int_{0}^{t^{\prime}} d \theta^{\prime} } \\
& \times \int_{0}^{\infty} k_{\mathrm{Rs}^{\prime}} c_{\mathbf{R}}{ }^{\prime}\left(r^{\prime}, \theta^{\prime}\right) c_{\mathbf{s}}{ }^{\prime}\left(r^{\prime}, \theta^{\prime}\right) 2^{\alpha} \pi r^{\prime \alpha} d r^{\prime}
\end{aligned}
$$

The quantities $2 N_{\mathrm{R}_{2}} / N_{0}$ and $N_{\mathrm{R}} / N_{0}$ [obtained from Eqs. (35) and (36) by letting $t \rightarrow \infty]$ represent the fraction of the initial number of radicals which undergo the radical-radical and the radical-solute reactions, respectively. $2 N_{\mathbf{R}_{2}} / N_{0}$ will be called the fractional molecular yield and $N_{\mathrm{Rs}} / N_{0}$ the fractional radical yield.

The physical constants which entirely describe the system are seven: two diffusion coefficients $D_{\mathrm{R}}$ and $D_{\mathrm{S}}$, 


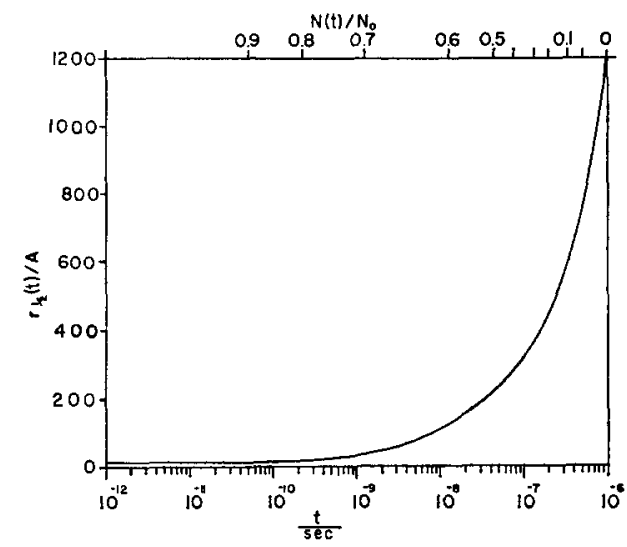

Fig. 5. Variation of $r_{1 / 2}(t)$ with time and fraction of unreacted radicals for a spherical spur. Gaussian initial distribution. Same parameters as in Fig. 1.

two second-order rate constants $k_{\mathrm{RR}}$ and $k_{\mathrm{RS}}$, the initial solute concentration $c_{\mathrm{s}_{0}}$, the initial spur or track radius $r_{0}$, and the radical-density parameter $N_{0}$. However, it follows from the preceding considerations that once the shape of the initial distribution is chosen (Gaussian, rectangular, etc.), the relative yields depend only on four quantities: the two dimensionless rate constants, $k_{\mathrm{RR}}{ }^{*}$ and $k_{\mathrm{Rs}}{ }^{*}$, the ratio $c_{\mathrm{s}_{0}}{ }^{*}$ (at the center of the spherical spur or axis of the cylindrical track) of solute to radical concentration, and the ratio $D_{\mathrm{s}}{ }^{\prime}$, of the diffusion coefficient of the solute to that of the radicals.

When it is possible to neglect the effect of solute depletion (see Sec. 4), Eq. (2) is not necessary (thus $D_{\mathrm{s}}{ }^{\prime}$ does not appear) and $c_{\mathrm{s}}$ is constant (independent of position and time). The fractional yields depend then only on the two dimensionless parameters $k_{\mathrm{RR}}{ }^{*}$ and $k_{\mathrm{RS}}{ }^{*} c_{\mathrm{S}_{0}}{ }^{*}$.

In Eqs. (26), (27), and (29) the dimensionless parameters were expressed in terms of $c_{R}(0,0)$. It is convenient to express them also in terms of $N_{0}$, by using Eq. (12). There results

$$
\begin{aligned}
& k_{\mathrm{RR}}{ }^{*}=[1 / f(\alpha, \beta)]\left(k_{\mathrm{RR}} N_{0} / D_{\mathrm{R}} r_{0}^{\alpha-1}\right), \\
& k_{\mathrm{RS}}{ }^{*}=\left[1 / f(\alpha, \beta)\left(k_{\mathrm{RS}} N_{0} / D_{\mathrm{R}} r^{\alpha-1}\right),\right. \\
& c_{\mathrm{B}_{0}}{ }^{*}=f(\alpha, \beta)\left(c_{\mathrm{S}_{0}} r_{0}^{\alpha+1} / N_{0}\right), \\
& k_{\mathrm{RS}}{ }^{*} c_{\mathrm{S}_{0}}{ }^{*}=k_{\mathrm{RS}} c \mathrm{~S}_{0} r_{0}{ }^{2} / D_{\mathrm{R}} \text {. }
\end{aligned}
$$

When solute depletion may be neglected, there exists a range of values of $k_{\mathrm{Rs}}{ }^{*} c_{\mathrm{S}_{0}}{ }^{*}$ in which the relative yields are independent of this parameter (see Sec. 4). For this range, yields depend exclusively on the single parameter $k_{\mathrm{RR}_{\mathrm{R}}}{ }^{*}$, which involves four of the constants which describe the system. Notice [from Eq. (37)] that then spherical spurs of different $N_{0}$ but identical $N_{0} / r_{0}$ will have the same fractional yields. For cylindrical tracks $(\alpha=1)$, the fractional yields (in this range of $\left.k_{\mathrm{Rs}}{ }^{*} c_{\mathrm{B}_{0}}{ }^{*}\right)$ do not depend on $r_{0}$ at all.

\subsection{Space-Time History of Spherical Spurs and Cylindrical Tracks}

The system of equations consisting of Eqs. (23) and (24) was integrated numerically on Illiac for a variety of initial distribution parameters, rate constants, and diffusion coefficients. Methods, accuracies, and computation times are described in Sec. 4 of paper I. In addition, the quantities $N(t) / N_{0}, 2 N_{\mathrm{R}_{2}}(t) / N_{0}$ and $N_{\mathbf{R}}(t) / N_{0}$ were calculated. In this section we will describe the space-time history of particular spherical spurs and axially homogeneous cylindrical tracks in order to convey a feeling for the orders of magnitudes of the times and dimensions involved.

\subsubsection{Spherical Spurs}

In Fig. 1 the quantities $N(t) / N_{0}, 2 N_{\mathbf{R}_{2}}(t) / N_{0}$ and $N_{\mathrm{R}}(t) / N_{0}$ are plotted against $\mathrm{t}^{4} \log (t / \mathrm{sec})$, for an initially Gaussian spherical spur. Parameters are $N_{0}=12$ radic, ${ }^{5} r_{0}=10 \mathrm{~A}, D_{\mathrm{R}}=4 \times 10^{-5} \mathrm{~cm}^{2} / \mathrm{sec}, D_{\mathrm{S}}=$ $4 \times 10^{-6} \mathrm{~cm}^{2} / \mathrm{sec}, \quad k_{\mathrm{RR}}=k_{\mathrm{RS}}=10^{-11} \mathrm{~cm}^{3} /(\mathrm{sec} \times \mathrm{radic})$ and $c_{\mathrm{S}_{0}}=10^{-3} \mathrm{~mole} / \mathrm{l}$. Because of Eqs. (37) through (39) the same results would be obtained for $N_{0}=6$ radic, $r_{0}=5 \mathrm{~A}$ and $c_{\mathrm{S}_{0}}=4 \times 10^{-3} \mathrm{~mole} / \mathrm{l}$, the other parameters being unchanged. Notice that at about $t=10^{-8} \mathrm{sec}$ the radical recombination reaction is practically over, whereas the radical-solute reaction has barely started; the latter is essentially over at about $t=10^{-6} \mathrm{sec}$. Thus the chemical history of the spur terminates in about $1 \mu \mathrm{sec}$. Furthermore, the radical-radical and radical-solute reactions do not overlap in time. However, this is not the case for higher solute

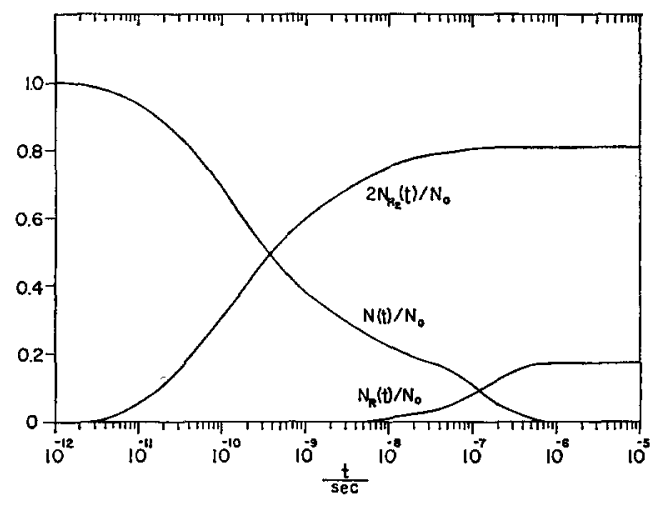

FIG. 6. Variation of $N(t) / N_{0}, 2 N_{\mathrm{R}_{2}}(t) / N_{0}$ and $N_{\mathrm{R}}(t) / N_{0}$ with time for an axially homogeneous cylindrical track. Gaussian initial distribution. $N_{0}=8.5 \times 10^{7} \mathrm{radic} / \mathrm{cm}, r_{0}=10 \mathrm{~A}, D_{\mathrm{R}}=$ $4 \times 10^{-5} \mathrm{~cm}^{2} / \mathrm{sec}, D_{\mathrm{s}}=4 \times 10^{-6} \mathrm{~cm}^{2} / \mathrm{sec}, k_{\mathrm{RR}}=k_{\mathrm{RS}}=10^{-11} \mathrm{~cm}^{3} /$ (sec $\times$ radic), $c_{\mathrm{s}_{0}}=10^{-8} \mathrm{~mole} / 1$.

4 We use $\log (t / \mathrm{sec})$ rather than $\log t$ in order to refer to the logarithm of a pure number, thus avoiding the necessity of defining the logarithm of a dimensional quantity.

${ }^{5}$ The symbol radic is defined in this paper as a unit of measurement of quantity of radicals. Thus, $N$ is expressed in radic and $k$ in $\mathrm{cm}^{8} /$ (sec $\times$ radic). This has the advantage of permitting a check of the correctness of the dimensionalities of the equations involved. To obtain $k$ in the more usual unit $1 /(\sec \times$ mole) one uses the relation $1 \mathrm{~cm}^{3} /(\mathrm{sec} \times$ radic $)=6.02 \times 10^{20} \mathrm{l} /(\mathrm{sec} \times \mathrm{mole})$. 
concentrations. For example, notice the results plotted in Fig. 2, which were obtained by making the initial solute concentration equal to $10^{-1}$ mole/ 1 rather than $10^{-3} \mathrm{~mole} / \mathrm{l}$, the other parameters being the same as for Fig. 1. It is seen that both the radical-radical and the radical-solute reactions occur concurrently to an appreciable extent and consequently that there is a competition between these two reactions which was almost lacking in the $10^{-3} \mathrm{~mole} / \mathrm{l}$ case. The competition decreases the value of $2 N_{\mathrm{R}_{2}} / N_{0}$. This decrease of molecular yields with increasing solute concentration will be further considered in Sec. 4 .

In Fig. 3 the radical and solute concentrations are plotted against the distance $r$ from the center of the spur for several values of $t$. Notice that there is a partial decrease of solute concentration in the center of the spur. Actually, this depletion is temporary. The

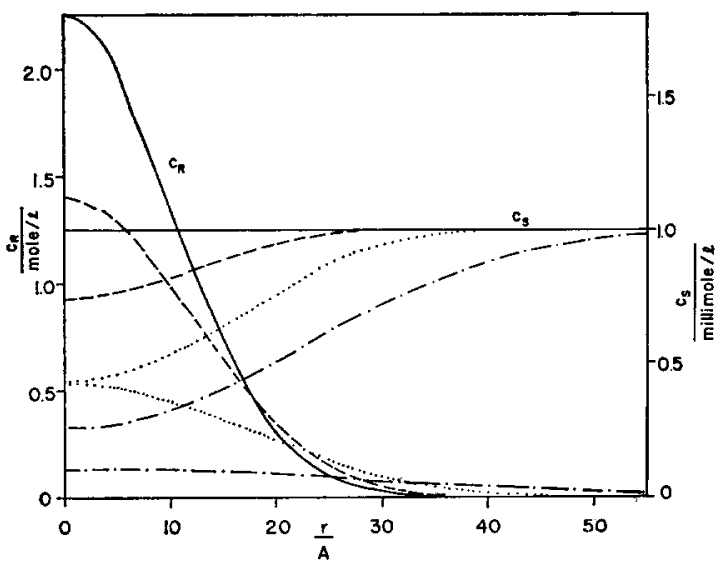

FIG. 7. Variation of $c_{\mathrm{R}}(r, t)$ and $c_{\mathrm{S}}(r, t)$ with $r$ for an axially homogeneous cylindrical track. Gaussian initial distribution. Same parameters as for Fig. 6. $t=0, N(t) / N_{0}=1$; $t=2.8 \times 10^{-11} \mathrm{sec}, N(t) / N_{0}=0.86 ; \cdots t=1.4 \times 10^{-10} \mathrm{sec}, N(t) /$ $N_{0}=0.64 ; \cdot \cdots \cdot t=5.9 \times 10^{-10} \mathrm{sec}, N(t) / N_{0}=0.44$.

minimum value $c_{\mathrm{s}}$ assumes throughout the life of this spur occurs at $t=5.9 \times 10^{-10} \mathrm{sec}$ (the largest time for which plots are given in Fig. 3). After this time diffusion of solute into the spur region starts building the concentration up to its initial value. In Fig. 4 the concentrations $c_{\mathrm{R}}(0, t)$ and $c_{\mathrm{S}}(0, t)$ at the center of the spur are plotted against time for the entire lifetime of the spur. This figure shows the minimum in the solute concentration referred to above.

In Fig. 5 the half-radius $r_{\frac{1}{2}}(t)$ (defined in Sec. 2.1) is plotted against time and $N(t) / N_{0}$ (upper scale). The parameters are the same as those for Fig. 1. The value of $2 N_{\mathrm{R}_{2}} / N_{0}$ is 0.366 , which means that after all reactions are over $36.6 \%$ of the radicals underwent the radical-radical reaction. Note that an appreciable fraction of this reaction is over before the spur has had time to expand very much. At $t=6.0 \times 10^{-9} \mathrm{sec}, 93.7 \%$ of the radical-radical reaction is over (as seen in Fig. 1), $N(t) / N_{0}=0.631$ and $r_{1}(t)=84 \mathrm{~A}$. Ninety-five percent of the radicals still unreacted at this time (corre-

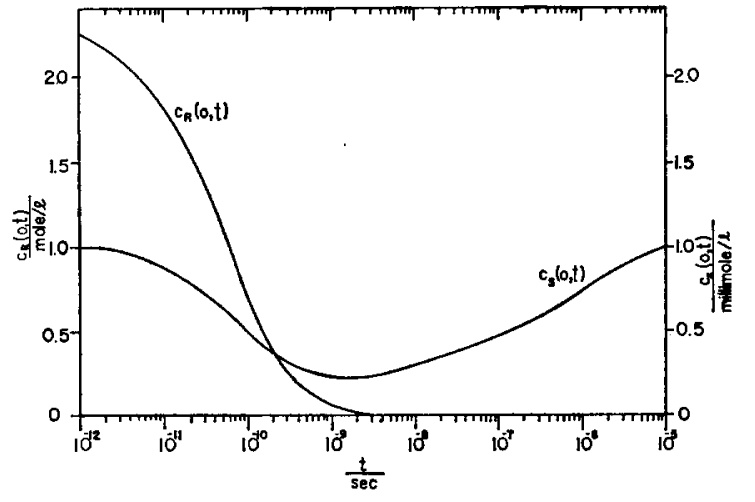

FIG. 8. Variation of $c_{\mathrm{R}}(0, t)$ and $c_{\mathrm{S}}(0, t)$ with time for an axially homogeneous cylindrical track. Gaussian initial distribution. Same parameters as for Fig. 6.

sponding to $60 \%$ of the initial number of radicals) are contained in a sphere whose radius is about $200 \mathrm{~A}$ [this can be calculated from the computed values of $\left.c_{\mathrm{R}}(r, t)\right]$. Therefore, for the conditions we have considered, there can be practically no spur interaction (i.e., reaction between radicals of different spurs) between spurs whose centers are more than $400 \mathrm{~A}$ apart.

\subsubsection{Axially Homogeneous Cylindrical Tracks}

An axially homogeneous cylindrical track is considered in Figs. 6 through 9. They correspond respectively to Figs. 1, 3, 4, and 5 except that $N_{0}$, instead of being $12 \mathrm{radic}$, is $8.5 \times 10^{7} \mathrm{radic} / \mathrm{cm}$. Because of Eqs. (34) through (36), the same results would be obtained for $r_{0}=5 \mathrm{~A}$ (instead of $10 \mathrm{~A}$ ) and $c_{\mathrm{s}_{0}}=4 \times 10^{-3} \mathrm{~mole} / 1$ (instead of $10^{-3} \mathrm{~mole} / \mathrm{l}$ ). This value of $N_{0}$ corresponds approximately to the linear radical density at the beginning of a $\mathrm{Po}^{210} 5.3 \mathrm{Mev} \alpha$-particle track, if $10 \mathrm{ev}$ is taken as the energy necessary to form a radical in liquid water.

These figures show that the time scale of events for this spur and track is about the same. The radical re-

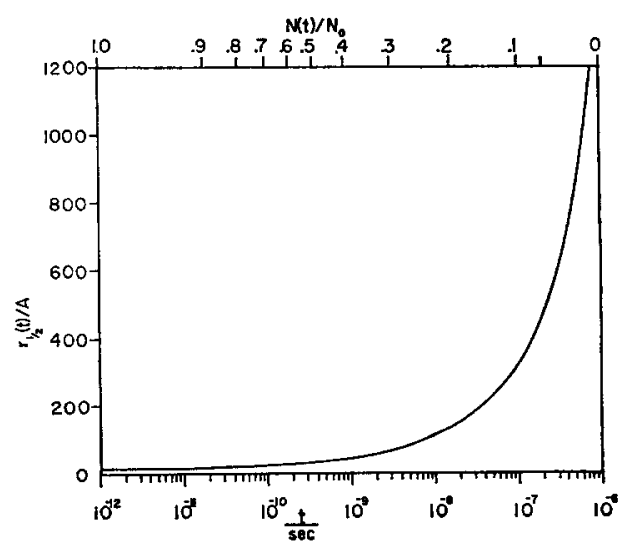

Fig. 9. Variation of $r_{1 / 2}(t)$ with time and fraction of unreacted radicals for an axially homogeneous cylindrical track. Gaussian initial distribution. Same parameters as for Fig. 6. 


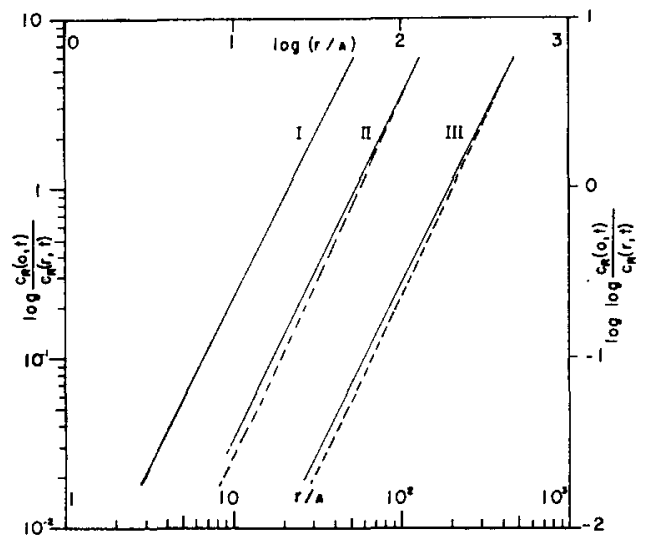

FIG. 10. Variation of $\log c_{\mathbf{R}}(0, t) / c_{\mathbf{R}}(r, t)$ with $r$. Gaussian initial distribution. $r_{0}=10 \mathrm{~A}, D_{\mathrm{R}}=4 \times 10^{-\mathrm{s}} \mathrm{cm}^{2} / \mathrm{sec}, D_{\mathrm{B}}=\infty$ $\left[c_{\mathrm{B}}(r, t)=c_{\mathrm{B}_{0}}\right], k_{\mathrm{RR}}=k_{\mathrm{Rs}}=10^{-11} \mathrm{~cm}^{3} /$ (sec $\times$ radic),$c_{\mathrm{S}_{0}}=10^{-8} \mathrm{~mole} / 1$. Spherical spur, $N_{0}=12$ radic; .-. Axially homogeneous cylindrical track, $N_{0}=8.5 \times 10^{7} \mathrm{radic} / \mathrm{cm}$. Curve I, $t=0, N(t) /$ $N_{0}=1, r_{1 / 2}=11.8 \mathrm{~A}$; Curves II, $t=5.9 \times 10^{-10} \sec N(t) / N_{0}=$ 0.733 (sph.), 0.440 (cyl.), $r_{1 / 2}(t)=30 \mathrm{~A}$ (sph.), $32 \mathrm{~A}$ (cyl.) Curves III, $t=9.6 \times 10^{-9} \mathrm{sec} N(t) / N_{0}=0.609$ (sph.), 0.158 (cyl.), $r_{1 / 2}(t)=106 \mathrm{~A}$ (sph.), $114 \mathrm{~A}$ (cyl.).

combination reaction is, however, slightly slower for the track than for the spur. It follows that the overlap in time between the radical recombination reaction and the radical-solute reaction is greater in the track than in the spur, as can be seen by comparing Figs. 1 and 6 . It is thus expected that, at these relatively low solute concentrations, the effect of $c_{\mathrm{s}_{0}}$ on the molecular yield should be larger for cylindrical tracks than for spherical spurs, other conditions being equal. This is indeed found to be the case. This topic will be further considered in Sec. 4.

The main difference between results for the cylindrical track and those for the spherical spur lies in the relative extents of the radical-radical and radicalsolute reactions. The value of $2 N_{\mathrm{R}_{2}} / N_{\mathrm{R}}$ is 0.58 for the spherical case and 4.32 for the cylindrical one. Thus the radical-radical reaction is greatly enhanced in cylindrical tracks. This enhancement is due to the higher radical density for cylindrical tracks as compared to equivalent spherical spurs. (By "equivalent spurs" we mean spurs such that the track can be assumed to be composed of a colinear string of them; see Sec. 6.3.) Indeed, the initial radical concentration along the axis of the track is 2.25 mole $/ 1$ whereas it is 1.26 mole/l at the center of the spur, as can be calculated from Eqs. (12) and (13).

We see from Fig. 6 that the value of $2 N_{\mathrm{R}_{2}} / N_{0}$ for the cylindrical track is 0.812 . At $t=9.6 \times 10^{-9} \mathrm{sec}$, $92.5 \%$ of the radical-radical reaction is over, $N(t) / N_{0}=$ 0.232 , and $r_{1}(t)=112$ A. $95 \%$ of the radicals still unreacted at this time (corresponding to $22 \%$ of the initial number of radicals) are contained in a cylinder of radius $228 \mathrm{~A}$. The interaction of two parallel tracks [of the kind considered in Figs. 5 through 9] whose axes are at least $456 \mathrm{~A}$ apart will, therefore, be negligible.

\section{VALIDITY OF THE PRESCRIBED DIFFUSION APPROXIMATION}

Prior to the use of digital computers in studying track effects in radiation chemistry, analytical methods were used ${ }^{1,6}$ to obtain approximate expressions for the molecular and radical yields. These methods were quite successful in explaining experimental results. The availability of numerical solutions of Eqs. (1) and (2) now permits a direct examination of the validity of the approximations used in those analytical treatments. These approximations were essentially two: (a) the "lack-of-solute-depletion" hypothesis and (b) the "prescribed diffusion" hypothesis.

The lack-of-solute-depletion hypothesis states that the solute concentration $c_{\mathrm{S}}(r, t)$ can be considered independent of time or position and thus constantly equal to its initial value $c_{\mathrm{s}_{0}}$. This approximation was used not only in all the analytical treatments but also in the first digital computer treatment of the one-radical model. ${ }^{7}$ The validity of this hypothesis will be examined in Sec. 4.

The prescribed diffusion hypothesis assumes, implicitly, that the lack-of-solution-depletion hypothesis is correct and, in addition, that: (1) the radial distribution of radicals in a spherical spur or axially homogeneous cylindrical track is initially Gaussian, and continues to be Gaussian as the spur or track expands, in spite of the reactions that go on; (2) the law of variation of the radius of this distribution with time is the same as if only diffusion were occurring. These approximations were introduced for the first time by $\mathrm{Jaffe}^{8}$ to explain the ionization currents produced in gases by ionizing radiation. $\mathrm{Lea}^{9,10}$ first applied them to the radiolysis of aqueous solutions.

We will examine first the validity of the prescribed diffusion approximation, assuming that the lack-ofsolute-depletion hypothesis holds; i.e., that only the first equation of the system of Eqs. (1) and (2) need

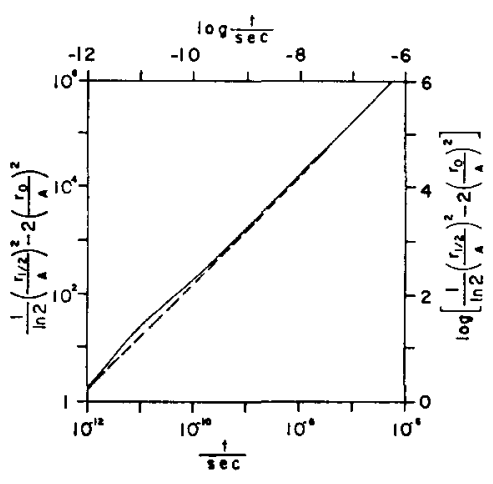

FIG. 11. Variation of $\left(r_{1 / 2}^{2} / \ln 2\right)-2 r_{0}^{2}$ with $\ell$ for a spherical spur. Gaussian initial distribution. Same parameters as in Fig. 10.

Numerical integration; --- Prescribed diffusion.

${ }^{6}$ See references $7,8,9$, and 21 of paper $I$.

7 D. A. Flanders and H. Fricke, J. Chem. Phys. 28, 1126 (1958).

${ }^{8}$ G. Jaffe, Ann. Physik, Ser. 4 42, 303 (1913).

$\because$ D. E. Lea, Actions of Radiations on Living Cells (Cambridge University Press, Cambridge, England, 1946).

${ }^{10}$ D. E. Lea, Brit. J. Radiol., Suppl. 1, 59 (1947). 
be considered, and that in it $c_{\mathrm{B}}$ is a constant equal to $c_{\mathrm{S}_{0}}$. We will, therefore, investigate to what extent the numerical solutions of the equation

$$
\partial c_{\mathrm{R}} / \partial t=D_{\mathrm{R}} \nabla^{2} c_{\mathrm{R}}-k_{\mathrm{RR}} c_{\mathrm{R}}^{2}-k_{\mathrm{RS}} c_{\mathrm{B}_{0}} c_{\mathrm{R}}
$$

(for Gaussian initial distributions) follow the prescribed diffusion assumption. This assumption can be quantitatively expressed by the relation

$$
c_{\mathbf{R}}(r, t)=c_{\mathbf{R}}(0, t) \exp \left[-r^{2} /\left(2 r_{0}^{2}+4 D t\right)\right] .
$$

From this there results

$$
\begin{aligned}
\log \frac{c_{\mathrm{R}}(0, t)}{C_{\mathrm{R}}(r, t)} & =\frac{\log 2}{\left[r_{\frac{1}{2}}(t)\right]^{2}}, \\
{\left[r_{\frac{3}{3}}(t)\right]^{2} } & =\left(2 r_{0}{ }^{2}+4 D t\right) \ln 2 .
\end{aligned}
$$

For testing purposes, we have considered a spherical spur and an axially homogeneous cylindrical track similar to those considered in the previous section.

FIG. 12. Variation of $\left(r_{1 / 2}^{2} / \ln 2\right)-2 r_{0}^{2}$ with $t$ for an axially homogeneous cylindrical track. Gaussian initial distribution. Same parameters as in Fig. 10. - _ Numerical integration; -- Prescribed diffusion.

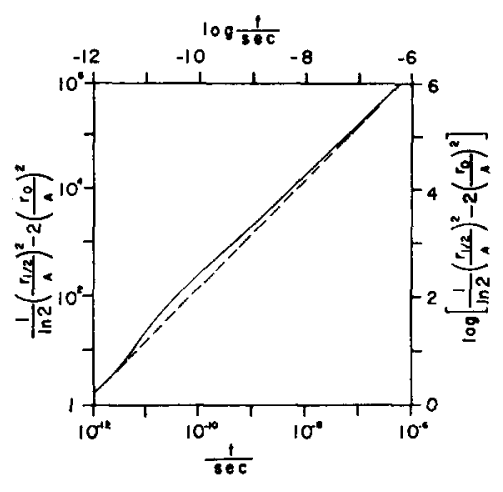

In Fig. 10, $\log \log \left[c_{\mathrm{R}}(0, t) / c_{\mathrm{R}}(r, t)\right]$ is plotted against $\log (r / \mathrm{A})$ for three values of time: $0,5.9 \times 10^{-10} \mathrm{sec}$ and $9.6 \times 10^{-9} \mathrm{sec}$. Values of $N(t) / N_{0}$ for these times are tabulated in the figure caption. $2 N_{\mathrm{R}_{2}} / N_{0}$ (at infinite time) is 0.366 for the spherical spur and 0.805 for the cylindrical track. A pertinent quantity is the extent to which the radical-radical reaction has proceeded at time $t$. This quantity can be measured by the ratio $\left[2 N_{\mathrm{R}_{2}}(t) / N_{0}\right] /\left[2 N_{\mathrm{R}_{2}} / N_{0}\right]$, which is equal, for the spherical spur, to $0,71.9 \%$, and $95.6 \%$ respectively (for the three times mentioned above) and, for the cylindrical track, to $0,68.9 \%$, and $93.0 \%$. The curves for $t=0$ (which coincide for the spur and track) are given just for comparison purposes, since the shape of the initial distribution is by hypothesis exactly Gaussian. Notice that all three curves for the spur are parallel straight lines with slope 2.00. This indicates that Eq. (43) is indeed satisfied and that, for the conditions considered, the Gaussian shape is preserved during the history of the spur. For the cylindrical track, curves II and III are slightly curved at low values of $r$ and straight lines of slope about 2.14 for large ones, indicating a larger deviation from Gaussian shape than that in the spherical case.

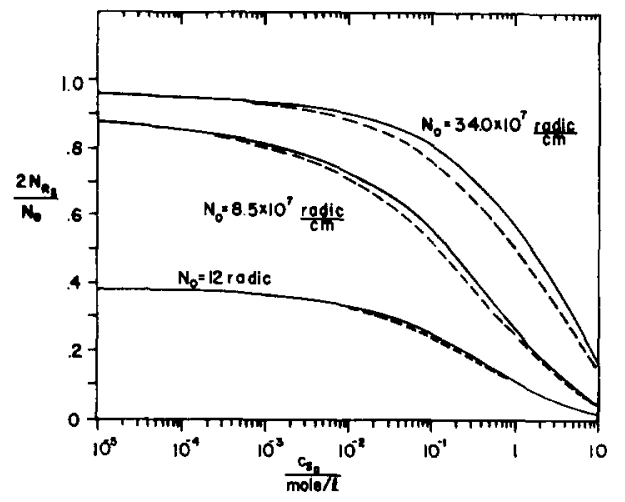

FIG. 13. Variation of $2 N_{\mathrm{R}_{2}} / N_{0}$ with $c_{\mathrm{B}_{0}}$ for a spherical spur and two axially homogeneous cylindrical tracks. Gaussian initial distributions. $r_{0}=10 \mathrm{~A}, D_{\mathrm{R}}=4 \times 10^{-6} \mathrm{~cm}^{2} / \mathrm{sec}, k_{\mathrm{RR}}=k_{\mathrm{Rs}}=10^{-11}$ $\mathrm{cm}^{3} /\left(\mathrm{sec} \times\right.$ radic) $.-D_{\mathrm{g}}=4 \times 10^{-6} \mathrm{~cm}^{2} / \mathrm{sec} ;-D_{\mathrm{B}} \rightarrow \infty$ (lackof-solute-depletion hypothesis).

In Figs. 11 and 12,

$$
\log \left[(1 / \ln 2)\left(r_{1} / A\right)^{2}-2\left(r_{0} / A\right)^{2}\right]
$$

is plotted against $\log (t / \mathrm{sec})$ for the spherical spur and cylindrical track. Also, as dashed lines, the prescribed diffusion curves, given by Eq. (44), are included. The latter are straight lines of slope 1.00 and intercept $\log \left[(4 D) /\left(\mathrm{A}^{2} / \mathrm{sec}\right)\right]$. It can be seen that the agreement between the digital computer results and the prescribed diffusion approximation is quite good, at least for times larger than about $5 \times 10^{-10} \mathrm{sec}$ for the spherical spur and $5 \times 10^{-9} \mathrm{sec}$ for the cylindrical track. We conclude that both Eqs. (43) and (44) are obeyed quite well for the spur and track considered, especially for the spur. However, small deviations do occur, principally during the period when the radical-radical reaction is proceeding. Actually, it is the occurrence of this reaction which causes these deviations, since if $k_{\mathrm{RR}}$ is zero, the prescribed diffusion approximation is exactly correct for Eq. (41). We may conclude that the fractional molecular yields $2 N_{\mathbf{R}_{2}} / N_{0}$ calculated using the prescribed diffusion approximation will be slightly smaller than the ones obtained from an integration of Eq. (41), this effect being more pronounced for cylindrical tracks



FIG. 14. Variation with initial solute concentration of relative error in $2 N_{\mathrm{R}_{2}} / N_{0}$ due to lack-of-solute-depletion hypothesis. 


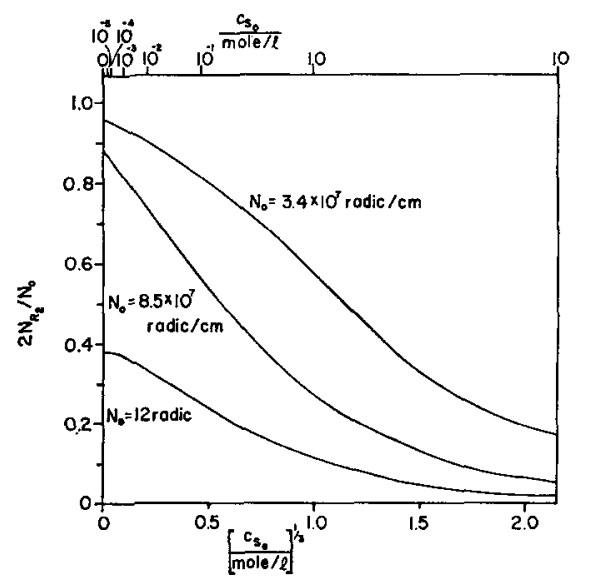

Fig. 15. Variation of $2 N_{\mathrm{R}_{2}} / N_{0}$ with $\left[c_{\mathrm{B}_{0}} /(\text { mole/l) }]^{1 / 3}\right.$ for the spur and tracks of Fig. 13. $D_{8}=4 \times 10^{-6} \mathrm{~cm}^{2} / \mathrm{sec}$.

than for spherical spurs. This is indeed found in general to be the case for the interesting ranges of parameters. ${ }^{7}$

\section{EFFECT OF SOLUTE CONCENTRATION AND DEPLETION ON YIELDS}

As we have pointed out in Secs. 2.2.1 and 2.2.2, it is expected in the diffusion model that the relative extents of the radical recombination and radical-solute reactions should decrease as the solute concentration increases.

It has also been pointed out that the solute concentration decreases temporarily in the region of the center of the spherical spur or axis of the cylindrical track, because of the radical-solute reaction (cf. Figs. $3,4,7$, and 8). Therefore, the lack-of-solute-depletion hypothesis is obviously incorrect. The main question, however, is how it affects the molecular and radical yields. It is expected that since this hypothesis produces a higher solute concentration than would otherwise result, it should enhance the effect of solute concentration on yields. Thus, a pertinent way of testing the importance of the lack-of-solute-depletion hypothesis is through its effect on the variation of yields with initial solute concentration.

A test of this kind is represented in Figs. 13 and 14. Notice that, as expected, the effect of this hypothesis is to decrease $2 N_{\mathrm{R}_{2}} / N_{0}$ below its correct value. Therefore, the relative error $\epsilon$ thus introduced in this yield is always negative. In Fig. 14, $-\epsilon$ is plotted against $c_{\mathbf{S}_{0}}$ for the spur and tracks considered.

Observation of Figs. 13 and 14 shows that for the spherical spur considered, the error in the fractional molecular yield $2 N_{\mathrm{R}_{2}} / N_{0}$ introduced by the lack-ofsolute-depletion hypothesis is very minor, never exceeding $5 \%$. Another way of stating this is to say that the calculated fractional yields are very insensitive to the value of the solute diffusion coefficient $D_{S}$, approximately the same results being obtained for $4 \times 10^{-6}$ $\mathrm{cm}^{2} / \mathrm{sec}$ and infinity. It is probably true that for practically all of the spherical spurs of interest the error introduced in the yields is relatively small. When, however, cylindrical tracks are considered, this error increases, becoming larger as the initial linear radical density increases. Thus, for $N_{0}=8.5 \times 10^{7} \mathrm{radic} / \mathrm{cm}$ the maximum error is $10 \%$ and for $N_{0}=3.4 \times 10^{8}$ radic/cm it is about $15 \%$. Consequently, if accuracies better than these are desired, it is best not to use this hypothesis for cylindrical tracks of high initial linear radical density. Recently Fricke and Phillips have obtained similar results."

At first sight the shapes of the curves of Fig. 14, showing maxima for some $c_{\mathbb{S}_{0}}$ between 1 and $10 \mathrm{~mole} / \mathrm{l}$, are somewhat surprising. It might be expected that, at low solute concentration, the solute might be strongly depleted in the center of the spur track for a long time, so that the effect of the lack-of-solute-depletion hypothesis on the yields might be large. However, at these low solute concentrations, the radical-radical and radical-solute reactions overlap only slightly in time (as mentioned in Secs. 2.2.1 and 2.2.2). Therefore, the main effect of solute depletion for these cases should be to make the radical-solute reaction take a little more time to reach completion, rather than to affect appreciably its extent. This is indeed found to be the case. Once this point has become clear, it is easy to understand why the maxima occur. The reason is that, at very high solute concentrations, the maximum extent of the solute depletion should be an ever decreasing fraction of the initial solution concentration, and thus $-\boldsymbol{\epsilon}$ should become increasingly smaller.

In going from $c_{\mathbb{S}_{0}}=10^{-5} \mathrm{~mole} / \mathrm{l}$ to $c_{\mathrm{S}_{0}}=10^{-4} \mathrm{~mole} / \mathrm{l}$, $2 N_{\mathrm{R}_{2}} / N_{0}$ decreases by 0.002 (from 0.378 to 0.376 ) for the spherical spur ( $N_{0}=12$ radic), and by 0.022 (from 0.879 to 0.857$)$ for the first cylindrical track $\left(N_{0}=\right.$ $\left.8.5 \times 10^{7} \mathrm{radic} / \mathrm{cm}\right)$. This larger effect of the solute concentration for the cylindrical track is due to the fact that in the latter the time overlap between the radical-radical and radical-solute reaction is more pro-

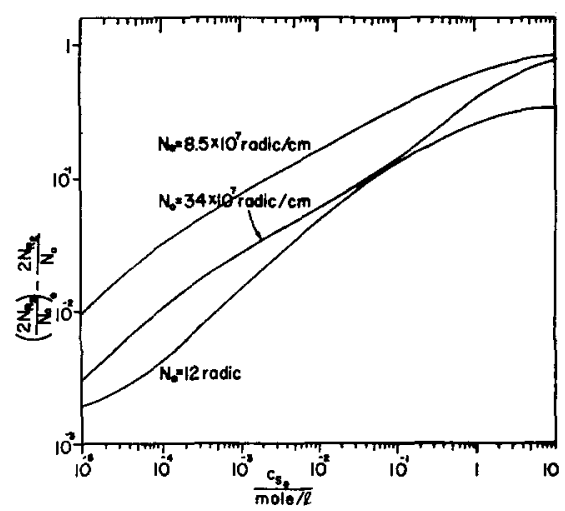

FIG. 16. Variation of $\log \left[\left(2 N_{\mathrm{R}_{2}} / N_{0}\right)_{0}-2 N_{\mathrm{R}_{2}} / N_{0}\right]$ with $\log \left[c_{\mathrm{S}_{0}} /\right.$ (mole/l) $]$ for the spur and tracks of Fig. 13. $D_{\mathrm{B}}=4 \times 10^{-6} \mathrm{~cm}^{2} / \mathrm{sec}$.

${ }^{11}$ H. Fricke and D. L. Phillips, J. Chem. Phys. 32, 1183 (1960). 34, 905 (1961). 
nounced than for the former, at least in the low-soluteconcentration region.

For reasons associated with the comparison of theory with experiment, it is convenient to plot $2 N_{\mathrm{R}_{2}} / N_{0}$ vs $\left[c_{\mathrm{S}_{0}} /(\mathrm{mole} / \mathrm{l})\right]^{1 / 3}$. This is done in Fig. 15. Notice that in certain intervals these curves can be approximated by straight lines. Thus, for the spherical spur, the fractional molecular yield $2 N_{\mathrm{R}_{2}} / N_{0}$ decreases linearly with the cube root of the concentration in the range of $10^{-4}$ to $0.5 \mathrm{~mole} / \mathrm{l}$. However, when $\log \left[\left(2 N_{\mathrm{R}_{2}} / N_{0}\right)_{0}-\right.$ $\left.2 N_{\mathrm{R}_{2}} / N_{0}\right]$ is plotted against $\log \left[c_{\mathrm{s}_{0}} /(\mathrm{mole} / \mathrm{l})\right]$, as in Fig. 16 [where $\left(2 N_{\mathrm{R}_{2}} / N_{0}\right)_{0}$ represents the value of $2 N_{\mathrm{R}_{2}} / N_{0}$ for $\left.c_{\mathrm{S}_{0}}=0\right]$, the curves obtained are not all straight lines with slope $\frac{2}{3}$. Nevertheless, in the solute concentration region $10^{-4}$ to $10^{-1} \mathrm{~mole} / \mathrm{l}$, the curves can be approximated by straight lines with slopes of 0.50 for $N_{0}=12$ radic (spherical case), 0.33 for $N_{0}=$ $8.5 \times 10^{7} \mathrm{radic} / \mathrm{cm}$ and 0.40 for $N_{0}=3.4 \times 10^{8} \mathrm{radic} / \mathrm{cm}$.

An interesting feature of Fig. 15 has to do with the value of $2 N_{\mathrm{R}_{2}} / N_{0}$ for $c_{\mathrm{S}_{0}}=0$, which represents the case of pure water. For the spherical case this value was obtained by solving the diffusion-kinetic equations up to sufficiently large values of $t$ for $2 N_{\mathrm{R}_{2}}(t) / N_{0}$ to have apparently reached an asymptotic value. In our case, for example, between $t=3.8 \times 10^{-7} \mathrm{sec}$ and $t=2.46 \times 10^{-6}$ sec, $2 N_{\mathbf{R}_{2}}(t) / N_{0}$ varied only between 0.378 and 0.379 . The latter was taken as $2 N_{\mathbf{R}_{2}} / N_{0}$. Actually $r_{\frac{1}{2}}$ at $t=$ $2.46 \times 10^{-6} \mathrm{sec}$ is already about $2750 \mathrm{~A}$, which is larger than the interspur distances of radiations of even very low LET. For example, the initial LET of $0.48 \mathrm{Mev}$ Compton recoil electrons typical of $\mathrm{Co}^{60} \gamma$ rays is about $0.021 \mathrm{ev} / \mathrm{A} .{ }^{9}$ Assuming an energy dissipation of about 50 to $100 \mathrm{ev}$ per spur, we obtain an interspur distance of about 2500 to $5000 \mathrm{~A}$. Consequently, for very large times, interaction between spurs occurs, a homogeneous radical distribution builds up, and back reactions of radicals with molecular products start. Therefore, even if in an infinite medium $2 N_{\mathrm{R}_{2}} / N_{0}$ approached unity (rather than 0.379), which is not excluded by these calculations (since in them it has not been possible to go beyond $t=2.46 \times 10^{-6} \mathrm{sec}$ and maintain adequate computational accuracy), it would not be pertinent to proceed with the calculation beyond that value of $t$. It is interesting to note, however, that the result $2 N_{\mathrm{R}_{2}} / N_{0}=0.379$ for $c_{\mathrm{B}_{0}}=0$ can also be obtained from Fig. 15 by the following process: Consider only that part of the curve for which $c_{\mathrm{S}_{0}} \geq 10^{-5} \mathrm{~mole} / 1$ and extrapolate this part back to $c_{\mathrm{s}_{0}}=0$. The agreement strongly indicates (but does not conclusively prove) that 0.379 is indeed the correct value in an infinite medium. The value obtained from the prescribed diffusion approximation, using the same parameters and the same diffusion kinetic equation ${ }^{12}$ is 0.402 .

For the cylindrical cases it was not possible to perform the calculation with $c_{\mathbb{S}_{0}}=0$ up to large enough values of $t$ for $2 N_{\mathrm{R}_{2}}(t) / N_{0}$ to become apparently con-

12 See reference 3, p. 103.



FIg. 17. Variation of $2 N_{\mathrm{R}_{2}} / N_{0}$ with $c_{\mathrm{B}_{0}} . D_{\mathrm{R}}=4 \times 10^{-6} \mathrm{~cm}^{2} / \mathrm{sec}$, $D_{\mathrm{S}}=4 \times 10^{-6} \mathrm{~cm}^{2} / \mathrm{sec}, k_{\mathrm{RR}}=k_{\mathrm{Rg}}=10^{-11} \mathrm{~cm}^{3} /(\mathrm{sec} \times$ radic $)$. Curves I: spherical spurs, $N_{0}=12$ radic. Curves II: axially homogeneous cylindrical tracks, $N_{0}=8.5 \times 10^{7} \mathrm{radic} / \mathrm{cm}$. Rectangular initial distribution, $r_{0}=15.55 \mathrm{~A}$; -- Gaussian initial distribution, $r_{0}=7.00 \mathrm{~A}$

stant, because the computational accuracy became very poor. However, back extrapolation to $c_{\mathrm{s} 0}=0$ of the curves in Fig. 15 furnishes $2 N_{\mathrm{R}_{2}} / N_{0}=0.884$ for $N_{0}=$ $8.5 \times 10^{7} \mathrm{radic} / \mathrm{cm}$ and $2 N_{\mathrm{R}_{2}} / N_{0}=0.960$ for $N_{0}=3.4 \times 10^{8}$ $\mathrm{radic} / \mathrm{cm}$. The values yielded by the prescribed diffusion approximation are unity. Just as in the case of spherical spurs, whether or not these limiting values are correct for infinite media is unimportant from the point of view of applying the results of these calculations to cylindrical tracks in pure water. However, at $t=$ $2.46 \times 10^{-6} \mathrm{sec}$, for the case of $N_{0}=8.5 \times 10^{7} \mathrm{radic} / \mathrm{cm}$ under consideration, the fraction of unreacted radicals $N(t) / N_{0}$ is 0.130 (as opposed to 0.621 for the spherical case) and the track half-radius is about $2900 \mathrm{~A}$. Thus, at such large times, there will be far fewer radicals unreacted in cylindrical tracks than in spherical spurs (regardless of whether in an infinite medium and at infinite time all radicals are reacted for one or both of these cases). Since homogeneous conditions are setting in by then, the total amount of back reaction induced by these leftover radicals will be smaller in the cylindrical case than in the spherical one. This results in a much larger net pure water decomposition by, let us say, $\mathrm{Po}^{210} 5.3 \mathrm{Mev} \alpha$ particles (cylindrical tracks) than by $\mathrm{Co}^{60} \gamma$ rays (spherical spurs).

\section{EFFECT OF SHAPE OF INITIAL DISTRIBUTION ON YIELDS}

As mentioned in Sec. 2.1 of paper I, Gaussian initial distributions of the primary species in spherical spurs or cylindrical tracks were arbitrarily assumed in all papers heretofore published dealing with track effects in the radiation chemistry of aqueous solutions. In order to test the importance of this assumption, calculations were made for rectangular initial distributions of the type defined by Eq. (6), both for spherical spurs and for axially homogeneous cylindrical tracks.

For the spherical case a calculation was performed with $r_{0}$ (the radius of the rectangular initial distribution) equal to $15.55 \mathrm{~A}$, the other parameters being the 


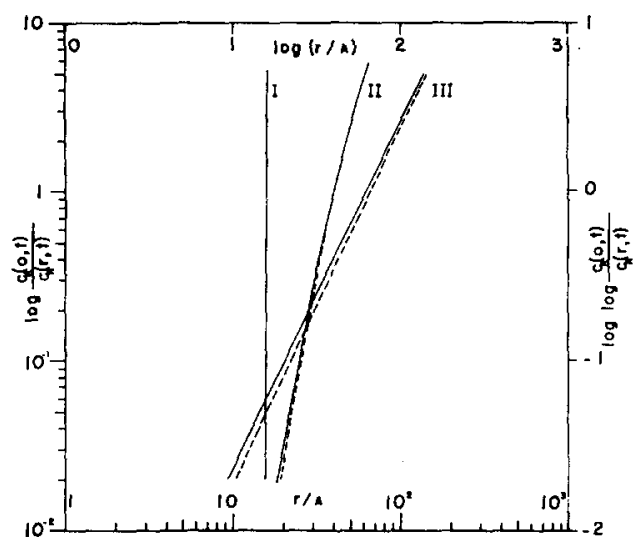

Fig. 18. Variation of $\log c_{\mathbf{R}}(0, t) / c_{\mathbf{R}}(r, t)$ with $r$. Rectangular initial distribution. $r_{0}=15.55 \mathrm{~A}, D_{\mathrm{R}}=4 \times 10^{-\mathrm{b}} \mathrm{cm}^{2} / \mathrm{sec}, D_{\mathrm{B}}=$ $4 \times 10^{-0} \mathrm{~cm}^{2} / \mathrm{sec}, k_{\mathrm{RR}}=k_{\mathrm{Rs}}=10^{-11} \mathrm{~cm}^{3} /(\mathrm{sec} \times \mathrm{radic}), c_{\mathrm{B}_{0}}=10^{-3}$ homogeneous cylindrical track, $N_{0}=8.5 \times 10^{7} \mathrm{radic} / \mathrm{cm}$. Curve I: $t=0, N(t) / N_{0}=1$. Curves II: $t=1.43 \times 10^{-11} \mathrm{sec}, N(t) / N_{0}=0.94$ (sph.), 0.89 (cyl.). Curves III: $t=9.27 \times 10^{-10} \mathrm{sec}_{1} N(t) / N_{0}=$ 0.62 (sph.), 0.35 (cyl.).

same as those of the spherical Gaussian spur of Fig. 1, for which $r_{0}=10 \mathrm{~A}$. The radical concentrations at the center of these two spurs is the same (and equal to $1.265 \mathrm{~mole} / \mathrm{l})$. The value of $2 N_{\mathrm{R}_{2}} / N_{0}$ calculated for the rectangular distribution is 0.456 , whereas for the Gaussian one it is 0.366 . This is understandable, since in the rectangular case the central radical concentration prevails up to $15.55 \mathrm{~A}$ away from the center, whereas in the Gaussian case the radical concentration has fallen to about $30 \%$ of its maximum value at this distance. Thus, the radical-radical reaction, in this region, should occur more rapidly in the former case than in the latter. Thus, if we wish a Gaussian spur furnishing the same value of $2 N_{\mathrm{R}_{2}} / N_{0}$ as the rectangular one being considered, we must make $r_{0}$ smaller than $10 \mathrm{~A}$ in order to have a value of $c_{\mathbb{R}}(0,0)$ larger than $1.265 \mathrm{~mole} / \mathrm{l}$. Actually, we found the required value of $r_{0}$ to be $7.00 \mathrm{~A}$ [and $\left.c_{\mathbf{R}}(0,0)=3.69 \mathrm{~mole} / 1\right]$, the other parameters being the same as those of the spur of Fig. 1. An interesting question is whether these two spurs will continue to furnish equal values of $2 N_{\mathrm{R}_{2}} / N_{0}$ as the solute concentration is varied. Calculations were performed for values of $c_{\mathrm{s}_{0}}$ between $10^{-5}$ and $10 \mathrm{~mole} / 1$ and the resulting values of $2 N_{\mathrm{R}_{2}} / N_{0}$ are plotted as curves I of Fig. 17. Notice that these two curves coincide except for very high solute concentrations, when the rectangular-case curve falls off a little more sharply than the Gaussian one.

Similar calculations were made for axially homogeneous cylindrical tracks with $N_{0}=8.5 \times 10^{7} \mathrm{radic} / \mathrm{cm}$. The curves II of Fig. 17, showing $2 N_{\mathrm{R}_{2}} / N_{0}$ as a function of $c_{\mathrm{S}_{0}}$, were obtained as in the spherical case, using $r_{0}=$ $15.55 \mathrm{~A}$ for the rectangular initial distribution and $r_{0}=7.00 \mathrm{~A}$ for the Gaussian one, the other parameters being the same as for the spherical case. Here also the agreement is excellent.
These results tend to indicate that changing over from Gaussian to rectangular initial distributions will result in a very minor change of the yields, as long as a new initial radius is adequately chosen.

An interesting question is whether a rectangular radical distribution approaches a Gaussian one as the spherical spur or cylindrical track expands. In Fig. 18 $\log \log \left[c_{\mathrm{R}}(0, t) / c_{\mathrm{R}}(r, t)\right]$ is plotted against $\log (r / \mathrm{A})$ for several values of $t$, for the spherical spur and axially homogeneous cylindrical track described above, with $c_{\mathrm{S}_{0}}=10^{-3} \mathrm{~mole} / \mathrm{l}$ in both cases. Notice that at about $t=10^{-9} \mathrm{sec}$ both have become almost exactly Gaussian. Most of the radical recombination (almost $80 \%$ of it) is over by then; it occurs mainly during the nonGaussian stage. But we have seen that, at least in the cases considered, this fact does not interfere with the dependence of yields on solute concentration.

\section{EFFECT OF RADICAL DENSITY, DIFFUSION COEFFICIENT AND RATE CONSTANTS ON YIELDS}

\subsection{Effect of Initial Radius and Initial Number of Radicals on Yields for Isolated Spherical Spurs}

Let us consider Eq. (28) and Eqs. (37) through (39), which express the four dimensionless parameters $D_{\mathrm{S}}{ }^{*}, k_{\mathrm{RR}}{ }^{*}, k_{\mathrm{RS}}{ }^{*}$, and $c_{\mathrm{S} 0}{ }^{*}$ in terms of $r_{0}$ and $N_{0}$. The first of them depends on neither $r_{0}$ nor $N_{0}$, the next two on $N_{0} / r_{0}$ (since $\alpha=2$ for spherical spurs) and the last one on $N_{0} / r_{0}^{3}$ [i.e., on $c_{\mathrm{R}}(0,0)$, as can be seen from Eq. (12)]. This suggests four ways of examining the effect of $N_{0}$ and $r_{0}$ on yields: (1) varying $r_{0}$ with $N_{0}$ constant; (2) varying $N_{0}$ with $r_{0}$ constant; (3) varying $N_{0}$ and $r_{0}$ with $N_{0} / r_{0}$ constant, and (4) varying $N_{0}$ and $r_{0}$ with $N_{0} / r_{0}{ }^{3}$ constant; i.e., $C_{\mathrm{R}}(0,0)$ constant.

The variation of $2 N_{\mathrm{R}_{2}} / N_{0}$ with $r_{0}, N_{0}$ being maintained constant, is depicted by curves I of Fig. 19. The solid line corresponds to a rectangular initial dis-

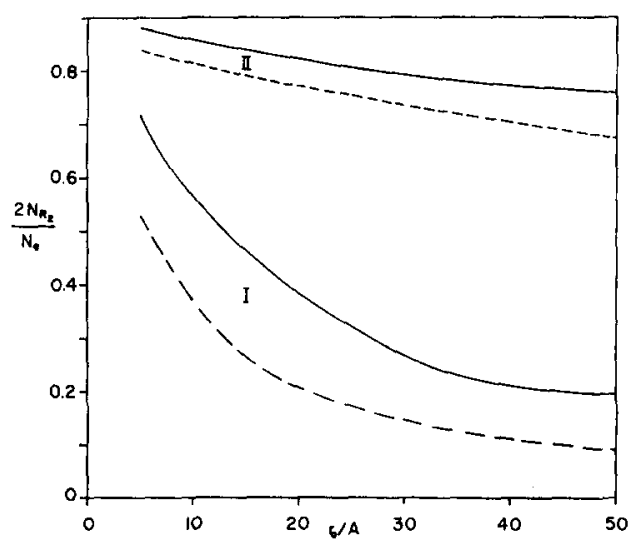

FIG. 19. Variation of $2 N_{\mathrm{R}_{2}} / N_{0}$ with $r_{0} . D_{\mathrm{R}}=4 \times 10^{-5} \mathrm{~cm}^{2} / \mathrm{sec}$, $D_{\mathrm{B}}=4 \times 10^{-6} \mathrm{~cm}^{2} / \mathrm{sec}, k_{\mathrm{RR}}=k_{\mathrm{RS}}=10^{-11} \mathrm{~cm}^{3} /(\mathrm{sec} \times \mathrm{radic}), c_{\mathrm{B}}=$ $10^{-2}$ mole/l. Curves I: spherical spurs, $N_{0}=12$ radic. Curves II: axially homogeneous cylindrical tracks, $N_{0}=8.5 \times 10^{7} \mathrm{radic} / \mathrm{cm}$. - Rectangular initial distribution; -.. Gaussian initial distribution. 
tribution and the dashed line to a Gaussian one. The parameters used are given in the figure caption. Notice that in the Gaussian case the decrease of $2 N_{\mathrm{R}_{2}} / N_{0}$ with $r_{0}$ is approximately linear for $r_{0}$ between 5 and $11 \mathrm{~A}$, the rate being about $0.031 / \mathrm{A}$. This rate slows down rapidly thereafter, falling to $0.0025 / \mathrm{A}$ by the time $r_{0}$ reaches $30 \mathrm{~A}$. The over-all shape of the curve for the rectangular initial distribution is similar to that for the Gaussian one, except that the former is shifted to the right. The reasons for this shift have been discussed in Sec. 5 .

The three previously mentioned ways of considering the variation of $2 N_{\mathrm{R}_{2}} / N_{0}$ with $N_{0}$ are represented by the curves of Fig. 20. The parameters chosen are given in the figure caption. Notice that the steepest increase of the fractional molecular yield $2 N_{\mathrm{R}_{2}} / N_{0}$ with $N_{0}$ is obtained by holding $r_{0}$ constant. If $N_{0} / r_{0}{ }^{3}$ is held constant, $2 N_{\mathrm{R}_{2}} / N_{0}$ increases in average a little more slowly with $N_{0}$. However, for fixed $N_{0} / r_{0}, 2 N_{\mathrm{R}_{2}} / N_{0}$ remains almost constant, actually decreasing slightly. The reason for this effect is that of the four dimensionless parameters $\mathrm{D}^{*}, k_{\mathrm{RR}}{ }^{*}, k_{\mathrm{Rs}}{ }^{*}$, and $c_{\mathrm{S}_{0}}{ }^{*}$, all but $c_{\mathrm{S}_{0}}{ }^{*}$ depend simply on $N_{0} / r_{0}$, and we have already seen in Sec. 4 that $2 N_{\mathrm{R}_{2}} / N_{0}$ is not a very sensitive function of $c_{\mathrm{S}_{0}}{ }^{*}$ for the values of $D_{\mathrm{R}}, k_{\mathrm{RR}}, k_{\mathrm{RS}}$, and $c_{\mathrm{S}_{0}}$ chosen. Parenthetically, it can be noted that changing $N_{0}$ and $r_{0}$ so that $N_{0} / r_{0}$ remains constant is equivalent to holding both $N_{0}$ and $r_{0}$ fixed and multiplying $c_{\mathrm{S}_{0}}$ by the square of the ratio of the new $r_{0}$ to the old one. This follows from the dependence of $c_{\mathrm{S}_{0}}{ }^{*}$ on $\left(r_{0}{ }^{3} / N_{0}\right)=$ $\left(r_{0} / N_{0}\right) r_{n}^{2}$.

\subsection{Effect of Initial Radius and Initial Linear Radical Density on Yields for Axially Homogeneous Cylindrical Tracks}

Again considering Eq. (28) and Eqs. (37) through (39), but with $\alpha=1$, we notice that $D_{\mathrm{s}}{ }^{*}$ depends neither on $r_{0}$ nor on $N_{0}, k_{\mathrm{RR}}{ }^{*}$ and $k_{\mathrm{Rg}}{ }^{*}$ depend on $N_{0}$ alone, and $c_{\mathrm{s}_{0}}{ }^{*}$ depends on $N_{0} / r_{0}{ }^{2}$; i.e., on $c_{\mathrm{R}}(0,0)$. This

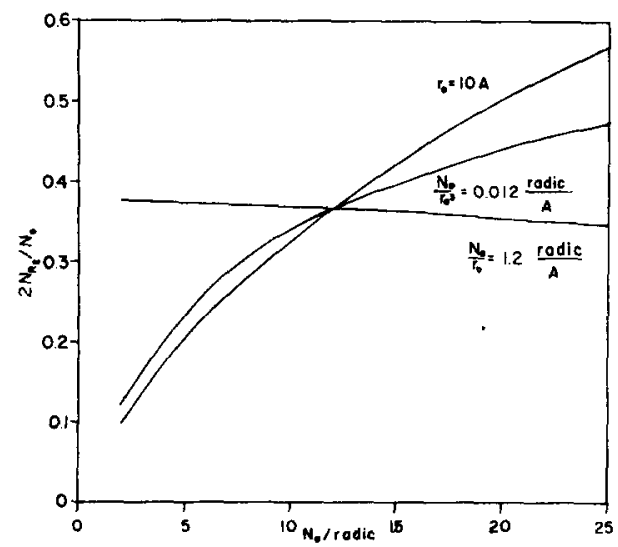

FIG. 20. Variation of $2 N_{\mathrm{R} 2} / N_{0}$ with $N_{0}$ for spherical spurs. Gaussian initial distribution. $D_{\mathrm{R}}=4 \times 10^{-5} \mathrm{~cm}^{2} / \mathrm{sec}, D_{8}=4 \times 10^{-1}$ $\mathrm{cm}^{2} / \mathrm{sec}, k_{\mathrm{RR}}=k_{\mathrm{Rg}}=10^{-11} \mathrm{~cm}^{3} /(\mathrm{sec} \times \mathrm{radic}), c_{\mathrm{B}_{0}}=10^{-3} \mathrm{~mole} / \mathrm{l}$.

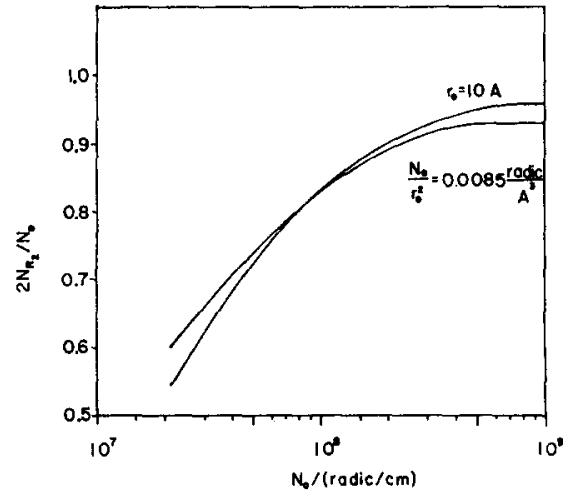

Fig. 21. Variation of $2 N_{\mathrm{R}_{2}} / N_{0}$ with $N_{0}$ for axially homogeneous cylindrical tracks. Gaussian initial distribution. $D_{R}=4 \times 10^{-5}$ $\mathrm{cm}^{2} / \mathrm{sec}, \quad D_{\mathrm{g}}=4 \times 10^{-6} \mathrm{~cm}^{2} / \mathrm{sec}, \quad k_{\mathrm{RR}}=k_{\mathrm{RS}}=10^{-11} \mathrm{~cm}^{2} /(\mathrm{sec} \times$ radic), $c_{\mathrm{B}_{0}}=10^{-8} \mathrm{~mole} / \mathrm{l}$.

suggests three ways of examining the effect of initial radius $r_{0}$ and initial linear radical density $N_{0}$ on yields: (1) varying $r_{0}$ with $N_{0}$ constant, (2) varying $N_{0}$ with $r_{0}$ constant, and (3) varying $N_{0}$ with $N_{0} / r_{0}^{2}$ constant; i.e., $c_{R}(0,0)$ constant.

The variation of $2 N_{\mathrm{R}_{2}} / N_{0}$ with $r_{0}, N_{0}$ being constant, is shown by curves II of Fig. 19, the solid line corresponding to a rectangular initial distribution and the dashed one to a Gaussian distribution. For both curves the initial linear radical density was $8.5 \times 10^{7} \mathrm{radic} / \mathrm{cm}$, the other parameters being the same as those for curves I (spherical spurs). It can be seen that for both the rectangular and the Gaussian initial conditions, the fractional molecular yield $2 N_{\mathrm{R}_{2}} / N_{0}$ varies very slowly with $r_{0}$. A fivefold change of $r_{0}$, from 10 to $50 \mathrm{~A}$, decreases $2 N_{\mathrm{R}_{2}} / N_{0}$ by only $10 \%$ for the rectangular case and $17 \%$ for the Gaussian one. The reason for this slow decrease is that $D_{\mathrm{s}}{ }^{*}, k_{\mathrm{RR}}{ }^{*}$, and $k_{\mathrm{Rg}}{ }^{*}$ are independent of $r_{0}$, whereas $c_{\mathrm{S}_{0}}{ }^{*}$ is proportional to $c_{\mathrm{s}_{0}}{ }^{2}{ }^{2} / N_{0}$. Therefore, changing $r_{0}$ to $r_{0}^{\prime}$ produces the same effect as fixing $r_{0}$ and changing the solute concentration from $c_{\mathrm{S}_{0}}$ to $c_{\mathbf{S}_{0}}{ }^{\prime}=\left(r_{0} / r_{0}\right)^{2} c_{\mathbf{s}_{0}}$. Since, as shown in Sec. 4, $2 N_{\mathrm{B}_{2}} / N_{0}$ is not a very sensitive function of $c_{\mathrm{S}_{0}}$ (between $10^{-5}$ and $10^{-2}$ mole $/ 1$ ) for the values of $D_{\mathrm{R}}, k_{\mathrm{RR}}, k_{\mathrm{Rs}}$, and $N_{0}$ chosen, it is expected that a fivefold change in $r_{0}$ (equivalent to a 25 -fold change in $c_{\mathrm{B}_{0}}$ ) should not affect $2 N_{\mathrm{R} 2} / N_{0}$ appreciably.

The two ways of studying the variation of $2 N_{\mathrm{R}_{2}} / N_{0}$ with $N_{0}$ are represented by the curves of Fig. 21. The parameters chosen are given in the figure caption. The two curves are very close to each other, which is a consequence of the fact (discussed in the preceding paragraph) that $2 N_{\mathrm{R}_{2}} / N_{0}$ varies very little with $r_{0}$ for the parameters chosen. Therefore, if the physico-chemical parameters $D_{\mathrm{R}}, D_{\mathrm{S}}, k_{\mathrm{RR}}, k_{\mathrm{Rs}}$, and $c_{\mathrm{S}_{0}}$ have known values close to the ones we have considered, $N_{0}$ can essentially be determined from a knowledge of the fractional molecular yield $2 N_{\mathrm{R}_{2}} / N_{0}$, even if $r_{0}$ is not known.

The variation of $2 N_{\mathrm{R}_{2}} / N_{0}$ with $N_{0}$ and $r_{0}, N_{0} / r_{0}^{2}$ and $c_{\mathrm{S}_{0}}$ being constant, is equivalent to its variation with 


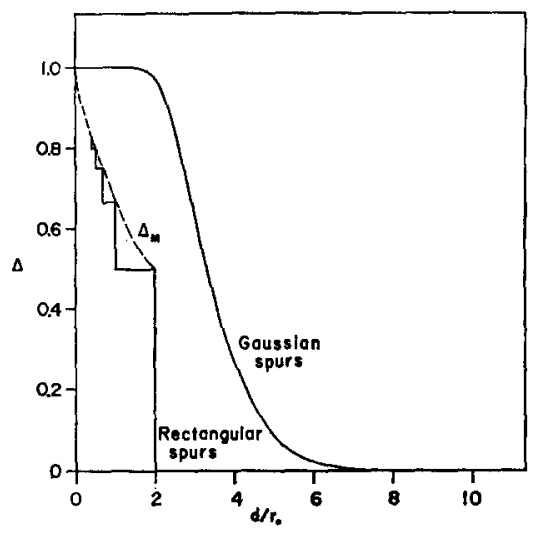

FIG. 22. Variation of $\Delta=\left[c_{\mathbf{R}}(r, z, 0)_{\min } / c_{R}(r, z, 0)_{\max }\right]$ (for constant $r$ ) with $d / r_{0}$ for an infinite colinear string of equidistant identical spherical spurs. $N_{0}=12$ radic, $D_{\mathrm{R}}=4 \times 10^{-5} \mathrm{~cm}^{2} / \mathrm{sec}$, $D_{\mathrm{s}}=4 \times 10^{-6} \mathrm{~cm}^{2} / \mathrm{sec}, k_{\mathrm{RR}}=k_{\mathrm{RS}}=10^{-11} \mathrm{~cm}^{3} / \mathrm{sec} \times$ radic $), c_{\mathrm{S}_{0}}=$ $10^{-3}$ mole $/ 1$.

$N_{0}$ and $c_{\mathrm{S}_{0}}, r_{0}$, and $c_{\mathrm{S}_{0}} / N_{0}$ being constant. Since $2 N_{\mathrm{R}_{2}} / N_{0}$ is not a sensitive function of $c_{\mathrm{S}_{0}}$, for the parameters chosen, most of the variation of the corresponding curve in Fig. 21 is due to the variation of $N_{0}$.

\subsection{Tracks as Strings of Equidistant Spherical Spurs}

As pointed out in Sec. 2.5, paper I, tracks are considered in one model to be strings of spherically symmetric spurs. Yields in such tracks have been studied analytically by Ganguly and $\mathrm{Magee}^{13}$ using the prescribed diffusion approximation. The case of isolated spherical spurs is attained when the spurs are initially so far apart that no interaction between them can occur. Axially homogeneous cylindrical tracks are formed when all spurs are equal and equidistant, have their centers aligned, and are initially so close together and in such a large number that they effectively merge into an axially homogeneous track whose length is very large compared to its diameter, making track-end effects negligible.

A pertinent question for comparison of the diffusionkinetic calculations with experiments has to do with the conditions under which these two extreme cases are applicable. These conditions can be estimated from the mathematical representation of such colinear strings of spurs. Let us choose a system of coordinates whose origin is the center of one spur and whose $z$ axis passes through the center of all spurs. Let the center of the $i$ th spur have a $z$ coordinate given by

$$
z_{i}=i d
$$

where $i$ is an integer and $d$ is the distance between the centers of neighboring spurs. Let $P(r, z)$ be a general point whose distance to the track axis is $r$. The generalized radical concentration (probability density) at

${ }^{13}$ A. K. Ganguly and J. L. Magee, J. Chem. Phys. 25, 129 13
A.
$(1956)$. point $P$ and time zero (as described in Sec. 2.2, paper I) is then

$$
c_{\mathrm{R}}(r, z, 0)=\sum_{i=-p}^{q} c_{\mathrm{R}}^{(i)}\left\{\left[r^{2}+(z-i d)^{2}\right]^{t}\right\},
$$

where $p+q+1$ is the total number of spurs in the track ( $p$ and $q$ being nonnegative integers) and $c_{R}^{(i)}$ is the contribution of the $i$ th spur to the radical concentration at point $P$. We will now consider the two cases of Sec. 2.1; i.e., Gaussian spurs and rectangular spurs.

\subsubsection{Gaussian Spurs}

The $i$ th spur contribution is given by

$$
c_{\mathrm{R}}{ }^{(i)}=c_{0} \exp \left\{-\left[r^{2}+(z-i d)^{2}\right] / 2 r_{0}{ }^{2}\right\} .
$$

The quantity $c_{0}$ is related to the initial number $N_{0}$ of radicals per spur and the initial spur radius $r_{0}$ by the expression

$$
c_{0}=N_{0} /(2 \pi)^{3 / 2} r_{0}{ }^{3} .
$$

The total concentration $c_{\mathbf{R}}$ is given by

$c_{\mathrm{R}}(r, z, 0)=\frac{N_{0}}{2 \pi^{3 / 2} r_{0}^{3}} \exp \left(-\frac{r^{2}}{2 r_{0}^{2}}\right) \sum_{i=-p}^{q} \exp \left[-\frac{(z-i d)^{2}}{2 r_{0}^{2}}\right]$.

Therefore, the radical distribution in any plane perpendicular to the track axis is Gaussian with the same initial radius $r_{0}$ which characterizes each spur. The variation of $c_{\mathrm{R}}$ along directions parallel to the track axis is entirely characterized by the function

$$
G(z)=\sum_{i=-p}^{q} \exp \left[-\frac{(z-i d)^{2}}{2 r_{0}^{2}}\right] .
$$

We assume that $d \neq 0$ and that $p$ and $q$ are sufficiently large so that for most of the track $G(z)$ can be replaced by the convergent series

$$
H(z)=\sum_{i=-\infty}^{\infty} \exp \left[-\frac{(z-i d)^{2}}{2 r_{0}^{2}}\right] .
$$

One thing we wish to determine is the range of $d / r_{0}$ over which the fluctuations in $H(z)$ are so small that $H(z)$ can be considered constant. It is obvious that $H(z)$ is periodic with period $d$ and can, therefore, be expanded in a Fourier series:

$H(z)=\frac{1}{2} a_{0}+\sum_{j=1}^{\infty} a_{j} \cos (2 \pi j z / d)+\sum_{j=1}^{\infty} b_{j} \sin (2 \pi j z / d)$,

where

$$
\left\{\begin{array}{l}
a_{j}=(2 / d) \int_{0}^{d} H(z) \cos \frac{2 \pi j z}{d} d z=\frac{(2 \pi)^{\frac{1}{2} r_{0}}}{d} \exp \left(-\frac{2 \pi^{2} r_{0}{ }^{2}}{d^{2}}\right) \\
b_{j}=(2 / d) \int_{0}^{d} H(z) \sin (2 \pi j z / d) d z=0
\end{array}\right.
$$


Substitution of (53) into (52) furnishes

$H(z)=\frac{(2 \pi) 2 r_{0}}{d}\left[\frac{1}{2}+\sum_{j=1}^{\infty} \exp \left(-\frac{2 \pi^{2} r_{0}^{2}}{d^{2}} j^{2}\right) \cos \frac{2 \pi j z}{d}\right]$.

By considering $d B / d z$ and $d^{2} H / d z^{2}$ we see that for $z=$ id ( $i$ integer) $H(z)$ has a maximum, that for $z=$ $\left(i+\frac{1}{2}\right) d, H(z)$ has a minimum, and that there are no other values of $z$ for which $H(z)$ is an extremum. Therefore, the ratio $\Delta$ between the lowest and highest values of $H(z)$ [and hence, under the approximations considered, of the lowest and highest $c_{\mathbb{R}}(r, z, 0)$ for constant $r]$ is

$$
\begin{aligned}
\Delta & =\frac{c_{\mathrm{R}}(r, d / 2,0)}{c_{\mathrm{R}}(r, 0,0)}=\frac{H(d / z)}{H(0)} \\
& =\frac{\frac{1}{2}+\sum_{j=1}^{\infty}(-1) j \exp \left(-\frac{2 \pi^{2} r_{0}^{2}}{d^{2}} j^{2}\right)}{\frac{1}{2}+\sum_{j=1}^{\infty} \exp \left(-\frac{2 \pi^{2} r_{0}^{2}}{d^{2}} j^{2}\right)} .
\end{aligned}
$$

The value of $\Delta$ depends only on $d / r_{0}$ and in Fig. 22 it is given as a function of this parameter by the uppermost curve. It was calculated using Eq. (55) for $d / r_{0} \leq 5$ and Eq. (51) for $d / r_{0}>5$, under which conditions the corresponding series converge very rapidly. Notice that for $d / r_{0} \leq 2$, the minimum value of $H(z)$ is larger than $97 \%$ of its maximum value. Therefore, in this range of $d / r_{0}$ the error in the calculated yields introduced by assuming $c_{\mathrm{R}}(r, z, 0)$ to be independent of $z$ is negligible. This permits us to establish a corresponding range of the average linear radical density $N_{d}$ in which this approximation is valid. Indeed, the relation between $N_{d}, N_{0}$, and $d$ is

$$
N_{d}=N_{0} / d \text {. }
$$

Thus, in order that $d / r_{0} \leq 2$ we must have

$$
N_{d} \geq N_{0} / 2 r_{0} \text {. }
$$

For our typical case of $N_{0}=12$ radic and $r_{0}=10 \mathrm{~A}$ (or $N_{0}=6$ radic and $\left.r_{0}=5 \mathrm{~A}\right) \mathrm{Eq}$. (57) furnishes $N_{d} \geq$ $6 \times 10^{7} \mathrm{radic} / \mathrm{cm}$. The maximum error introduced into $2 N_{\mathrm{R}_{2}} / N_{0}$ by assuming that in this range of $N_{d}$ we have an axially homogeneous cylindrical track can be estimated from Fig. 21. At $N_{d}=6 \times 10^{7} \mathrm{radic} / \mathrm{cm}$, for the curve corresponding to $r_{0}=10 \mathrm{~A}$, a change in $N_{d}$ of $3 \%$ (corresponding to the value of $1-\Delta$ for $d / r_{0}=2$ ) brings about a change in $2 N_{\mathrm{R}_{2}} / N_{0}$ of about $0.5 \%$. Actually, we can probably approximate an infinite string of colinear spherical Gaussian spurs by an axially homogeneous cylindrical track for lower values of $N_{d}$ than those given by Eq. (57), but an estimate of just how much lower requires solving the two-space-dimension diffusion-kinetic equations which describe the string of spurs being considered. This problem will be discussed in a future publication of this series.

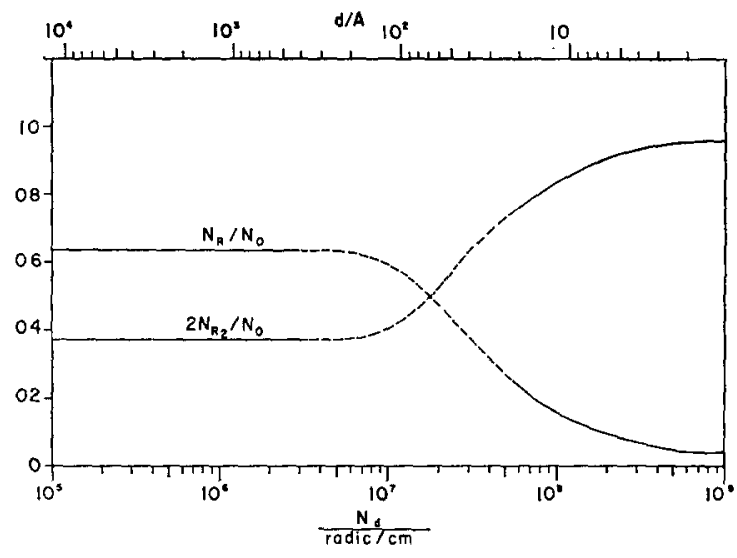

FIG. 23. Variation of $2 N_{\mathrm{R}_{2}} / N_{0}$ and $N_{\mathrm{R}} / N_{0}$ with initial average linear radical density $N_{d}$ and interspur distance $d$ for an infinite colinear string of equidistant identical spherical spurs. Same parameters as for Fig. 22.

Next, we wish to determine the value of $d / r_{0}$ above which the string of spherical Gaussian spurs can be replaced by isolated spurs. This value can be obtained by referring to the space-time history of such spurs. For example, it was seen in Sec. 2.2.1 that, for spurs of the type considered in Fig. 1 , when $d \geq 400 \mathrm{~A}$, there can be practically no spur interaction. However, an estimate of the small amount of spur interaction which does occur under these conditions requires once more a solution of the two-space-dimension problem.

The variation of the fractional molecular and radical yields with the initial average linear radical density $N_{d}$ or the initial interspur distance $d$ is plotted in Fig. 23 for an infinite colinear string of equidistant and identical spherical Gaussian spurs. The left-hand portions of the curves correspond to isolated spherical spurs $(d \geq 400$ A), the right-hand portions to axially homogeneous cylindrical tracks $\left(d \leq 2 r_{0}=20 \mathrm{~A}\right)$ and the center dashed portions, obtained by smoothly joining the other two, to the range of values of the inter-spur distance $(20 \mathrm{~A}<d<400 \mathrm{~A})$ for which the isolated spur or axially homogeneous cylindrical track approximations are not valid.

Finally, we may estimate the conditions under which track-end effects are negligible. A reasonable criterion is to require that the initial length of the track be much larger than the half-radius of a spur in which $95 \%$ of the radicals have disappeared. For the conditions of Fig. 1, this happens at about $t=4 \times 10^{-7} \mathrm{sec}$, when $r_{1}=660 \mathrm{~A}$ (see Fig. 5). Therefore, if the initial length of the track is, let us say, 50 times larger than that (about $33 \mu$ ), track-end effects should be less than a few percent. Hence to eliminate track-end effects, tracks such as those considered in Fig. 22, with $d=12$ A, must contain about 275 spurs.

We have neglected the fact that in a real track the average distance between successive spurs decreases as the high-energy particle generating the track slows 
down. This fact will be considered in a subsequent paper dealing with comparison with experiment.

\subsubsection{Rectangular Spurs}

The $i$ th spur contribution is given by

$$
c_{\mathrm{R}}^{(i)}=\gamma(\boldsymbol{r}) \boldsymbol{\gamma}_{i}^{\prime}(z) c_{0}
$$

where

$$
\gamma(r)=\left\{\begin{array}{l}
1 \text { for } r<r_{0}, \\
0 \text { for } r>r_{c}
\end{array}\right.
$$

and

$$
\gamma_{i}^{\prime}(z)=\left\{\begin{array}{l}
1 \text { for }|z-i d|<r_{0}, \\
0 \text { for }|z-i d|>r_{0} .
\end{array}\right.
$$

The quantity $c_{0}$ is related to the initial number $N_{0}$ of radicals per spur and the initial spur radius $r_{0}$ by the expression

$$
c_{0}=N_{0} / \frac{4}{3} \pi r_{0}^{3} \text {. }
$$

The total concentration $c_{\mathrm{R}}$ for an infinite string of rectangular spherical spurs is given by

$$
c_{\mathrm{R}}(r, z, 0)=\frac{N_{0}}{\frac{4}{3} \pi r_{0}{ }^{3}} \gamma(r) \sum_{i=-\infty}^{\infty} \gamma_{i}^{\prime}(z) \text {. }
$$

Here also the radical distribution in any plane perpendicular to the track axis is rectangular and has the same.initial radius as each individual spur. The function

$$
H^{\prime}(z)=\sum_{i=-\infty}^{\infty} \gamma_{i}^{\prime}(z)
$$

which describes the variation of $c_{R}$ along directions parallel to the track axis, is a periodic step function of $z$ of period $d$. For $[2 /(j+1)]<\left(d / r_{0}\right)<(2 / j)$, where $j$ is a positive integer, the smallest value of $H^{\prime}(z)$ in the interval $0<z<d$ is $j$, whereas its largest value is $j+1$. Therefore, the ratio $\Delta$ between the lowest and highest value of $H^{\prime}(z)$ [or $c_{\mathbb{R}}(r, z, 0)$, for $\left.r<r_{0}\right]$ is

$$
\Delta=\frac{\left[c_{\mathrm{R}}(r, z, 0)\right]_{\min }}{\left[c_{\mathrm{R}}(r, z, 0)\right]_{\max }}=\frac{\left[H^{\prime}(z)\right]_{\min }}{\left[H^{\prime}(z)\right]_{\max }}=\frac{j}{j+1} .
$$

In Fig. 22, $\Delta$ is given by the steplike curve. The smooth dashed curve passing through its edges represents the function

$$
\Delta_{M}=\left(1+d / 2 r_{0}\right)^{-1},
$$

which satisfies the relation $\Delta \leq \Delta_{M}$ for any $d / r_{0}$. As $2 r_{0} / d$ decreases from a nonintegral to an integral value, $\Delta$ approaches $\Delta_{M}$. It can be seen from Fig. 22 that a smaller value of $d / r_{0}$ (i.e., closer spurs) is required for rectangular spurs than for Gaussian ones to furnish the same value of $\Delta$. Since $\Delta$ is a measure of the axial homogeneity of the track, as the interspur distance decreases, the axially homogeneous approximation should become valid sooner for the Gaussian case than for the rectangular one. On the other hand, as seen in Sec. 6.2, Gaussian axially homogeneous tracks have smaller radii than rectangular ones with the same yields, the other independent parameters being equal. For example, a Gaussian track with $r_{0}=10 \mathrm{~A}$ furnishes the same yield as a rectangular one of radius $22 \mathrm{~A}$ (see Fig. 19). This partially compensates for the effect mentioned above, if we wish to compare tracks with equal yields. However, the magnitude of this effect can only be ascertained by an actual solution of the two-space-dimensional problem. If this effect is important, it might eventually furnish a means to determine the shapes of spurs.

\subsection{Effect of Radical Diffusion Coefficient and Rate Constants on Yields}

As seen in Sec. 2.1, yields are entirely determined by the four dimensionless parameters $D_{\mathrm{S}}{ }^{*}, k_{\mathrm{RR}}{ }^{*}, k_{\mathrm{RS}}{ }^{*}$ and $c_{\mathrm{S}_{0}}{ }^{*}$ given by Eqs. (28) and (37) through (39). Since we have already considered the effect on yields of the four independent parameters $c_{\mathrm{S}_{0}}$ (Sec. 4), $D_{\mathrm{S}}$ (Sec. 4), $r_{0}$ and $N_{0}$ (Secs. 6.1 and 6.2), it would seem that the effect of the remaining three parameters $\left(D_{\mathrm{R}}, k_{\mathrm{RR}}\right.$, and $k_{\mathrm{RS}}$ ) should be derivable from those. This is indeed the case for $D_{\mathrm{R}}$, but not for $k_{\mathrm{RR}}$ and $k_{\mathrm{RS}}$, as we shall show.

Let us change $k_{\mathrm{RR}}, k_{\mathrm{RS}}$, and $D_{\mathrm{R}}$ to $k_{\mathrm{RR}}{ }^{\prime}, k_{\mathrm{Rs}}{ }^{\prime}$ and $D_{\mathrm{R}}{ }^{\prime}$, respectively, maintaining the other parameters $D_{\mathrm{S}}, N_{0}$, $r_{0}$, and $c_{\mathrm{S}_{0}}$ unchanged. We wish to find new values $D_{\mathrm{s}}{ }^{\prime}, N_{0}{ }^{\prime}, r_{0}{ }^{\prime}$, and $c_{\mathrm{s}_{0}}{ }^{\prime}$ so that the set of physico-chemical parameters $k_{\mathrm{RR}}, k_{\mathrm{RS}}, D_{\mathrm{R}}, D_{\mathrm{S}^{\prime}}, N_{0}^{\prime}, r_{0}^{\prime} / c_{\mathrm{S}_{0}}$ furnish the same yields as the alternate set $k_{\mathrm{RR}^{\prime}}, k_{\mathrm{RS}^{\prime}}, D_{\mathrm{R}^{\prime}}, D_{\mathrm{S}}, N_{0}$, $r_{0}, c_{\mathbb{S}_{0}}$. For this to occur it is necessary, according to Eqs. (28) and (37) through (39), that

$$
\begin{aligned}
& D_{\mathrm{S}}{ }^{\prime} / D_{\mathrm{R}}=D_{\mathrm{s}} / D_{\mathrm{R}}{ }^{\prime}, \\
& k_{\mathrm{RR}} N_{0}^{\prime} / D_{\mathrm{R}} r_{0}{ }^{\alpha-1}=k_{\mathrm{RR}}{ }^{\prime} N_{0} / D_{\mathrm{R}}{ }^{\prime} r_{0}^{\alpha-1} \text {, } \\
& k_{\mathrm{RS}} N_{0}^{\prime} / D_{\mathrm{R}} r_{0}^{\prime \alpha-1}=k_{\mathrm{RS}}{ }^{\prime} N_{0} / D_{\mathrm{R}^{\prime}} r_{0}^{\alpha-1} \text {, } \\
& c_{\mathrm{S}_{0}}{ }^{\prime} r_{0}{ }^{\prime \alpha+1} / N_{0}{ }^{\prime}=c_{\mathrm{S}_{0} r_{0}{ }^{\alpha+1} / N_{0}} \text {. }
\end{aligned}
$$

Equations (66) through (69) are to be regarded as a system of four equations in the unknowns $D_{\mathbb{8}}{ }^{\prime}, N_{0}{ }^{\prime}$, $r_{0}^{\prime}$ and $c_{\mathrm{s}_{0}}{ }^{\prime}$, the other quantities being assumed known. Therefore, to obtain the effect of a variation of $D_{\mathrm{R}}$ to $D_{\mathrm{R}}{ }^{\prime}$, it is sufficient to calculate the yields for the parameters $k_{\mathrm{RR}}, k_{\mathrm{RS}}, D_{\mathrm{R}}, D_{\mathrm{S}^{\prime}}{ }^{\prime}, N_{0}{ }^{\prime}, r_{0}, c_{\mathrm{S}_{0}}{ }^{\prime}$, where

$$
\begin{aligned}
& D_{\mathrm{s}}{ }^{\prime}=\left(D_{\mathrm{R}} / D_{\mathrm{R}}{ }^{\prime}\right) D_{\mathrm{S}}, \\
& N_{0}{ }^{\prime}=\left(D_{\mathrm{R}} / D_{\mathrm{R}}{ }^{\prime}\right) N_{0}, \\
& c_{\mathrm{S}_{0}}{ }^{\prime}=\left(D_{\mathrm{R}} / D_{\mathrm{R}}{ }^{\prime}\right) c_{\mathrm{S}_{0} .}
\end{aligned}
$$

Consequently a change of $D_{\mathrm{R}}$ to $D_{\mathrm{R}}{ }^{\prime}$ is equivalent to holding $D_{\mathrm{R}}$ constant and multiplying $D_{\mathbf{s}}, N_{0}$, and $c_{\mathrm{S}_{0}}$ by $D_{\mathrm{R}} / D_{\mathrm{R}}{ }^{\prime}$. It was seen in Sec. 4 that the effect of $D_{\mathrm{S}}$ on yields was small. Therefore, the effect of a decrease in $D_{\mathbf{R}}$ is essentially the same as an equal relative increase in $N_{0}$ and $c_{\mathrm{S}_{0}}$. Since, for our parameters, yields 
also vary slowly with $c_{\mathrm{B}_{0}}$, the curve for $r_{0}=10 \mathrm{~A}$ in Fig. 20 can be considered to represent the variation of $2 N_{\mathrm{R}_{2}} / N_{0}$ with $1 / D_{\mathrm{R}}$ after the units of the horizontal axis are adequately changed. A similar statement is valid for the equivalent curve of Fig. 21. Consequently, the yields for spherical spurs are a much more sensitive function of $D_{\mathrm{R}}$ than those for cylindrical tracks. For instance, for the conditions of Fig. 20 (spherical spurs) and $N_{0}=12$ radic, as $D_{\mathrm{R}}$ changes from $4 \times 10^{-5} \mathrm{~cm}^{2} / \mathrm{sec}$ to $2 \times 10^{-5} \mathrm{~cm}^{2} / \mathrm{sec}, 2 N_{\mathrm{R}_{2}} / N_{\mathrm{0}}$ increases from about 0.366 to about 0.522 ; for the similar conditions of Fig. 21 (cylindrical tracks) and $N_{0}=8.5 \times 10^{7} \mathrm{radic} / \mathrm{cm}$, $2 N_{\mathrm{R}_{2}} / N_{0}$ varies from about 0.812 to about 0.888 for an equal change in $D_{\mathrm{R}}$.

The effect of changing $k_{\mathrm{RR}}$ to $k_{\mathrm{RR}}{ }^{\prime}$ cannot be obtained by simply changing $N_{0}, r_{0}, c_{\mathrm{S}_{0}}$ and $D_{\mathrm{s}}$. Indeed, in order that Eqs. (67) and (68) be compatible, it is necessary that

$$
k_{\mathrm{RR}} / k_{\mathrm{Rs}}=k_{\mathrm{RR}} / k_{\mathrm{RS}}
$$

Thus we cannot change $k_{\mathrm{RR}}$ without also changing $k_{\mathrm{RS}}$, if we wish to hold constant the values of the dimensionless parameters which determine the yields. Consequently, to determine the effect of $k_{\mathrm{RR}}$ on yields, it is necessary to make independent diffusion-kinetic calculations, varying $k_{\mathrm{RR}}{ }^{*}$ with the other dimensionless parameters $\left(k_{\mathrm{Rs}}{ }^{*}, D_{\mathrm{s}}{ }^{*}\right.$, and $\left.c_{\mathrm{S}_{0}}{ }^{*}\right)$ constant. Results of such calculations are given in Fig. 24. Notice that for the spherical case $2 N_{\mathrm{R}_{2}} / N_{0}$ increases almost linearly with

$$
\log \frac{k_{\mathrm{RR}}}{\mathrm{cm}^{3} /(\sec \times \text { radic })}
$$

but that for the cylindrical case the increase is slower. When solute depletion is unimportant, the effect of $k_{\mathrm{RR}}$ on yields can be obtained from the effect of $N_{0}$ on yields. Indeed, under these conditions Eq. (66) can be ignored $\left(D_{\mathrm{s}}=D_{\mathrm{s}}^{\prime}=0\right)$ and, because of Eq. (40), Eqs. (68) and (69) can be replaced by

$$
\left(k_{\mathrm{Rs}}{ }^{\prime} c_{\mathrm{s}_{0}}\right) r_{0}{ }^{2} / D_{\mathrm{R}}{ }^{\prime}=\left(k_{\mathrm{Rs}} c_{\mathrm{s}_{0}}{ }^{\prime}\right) r_{0}{ }^{\prime 2} / D_{\mathrm{R}} .
$$

It is now sufficient to satisfy Eqs. (67) and (74). This is achieved by making $r_{0}{ }^{\prime}=r_{0}, D_{\mathrm{R}}=D_{\mathrm{R}}{ }^{\prime}, \quad k_{\mathrm{Rs}}{ }^{\prime} c_{\mathrm{S}_{0}}=$ $k_{\mathrm{RS}} \mathrm{s}_{\mathrm{s}}{ }^{\prime}$ and

$$
N_{0}^{\prime}=\left(k_{\mathrm{RR}}^{\prime} / k_{\mathrm{RR}}\right) N_{0} .
$$

Fig. 24. Variation of $2 N_{\mathrm{R} g} / N_{0}$ with $k_{\mathrm{RR}}$ for Gaussian spherical spurs and axially homogeneous cylindrical tracks. $r_{0}=10 \mathrm{~A}, D_{\mathrm{R}}=4 \times 10^{-6}$ $\mathrm{cm}^{2} / \mathrm{sec}, \quad D_{\mathrm{s}}=4 \times 10^{-6}$ $\mathrm{cm}^{2} / \mathrm{sec}, \quad k_{\mathrm{Rg}}=10^{-11}$ $\mathrm{cm}^{2} /$ (sec $\times$ radic), $c_{\mathrm{s}}=$ $10^{-8}$ mole/1. Spherical case: $N_{0}=12$ radic. Cylindrical case: $N_{0}=$ $8.5 \times 10^{7} \mathrm{radic} / \mathrm{cm}$.

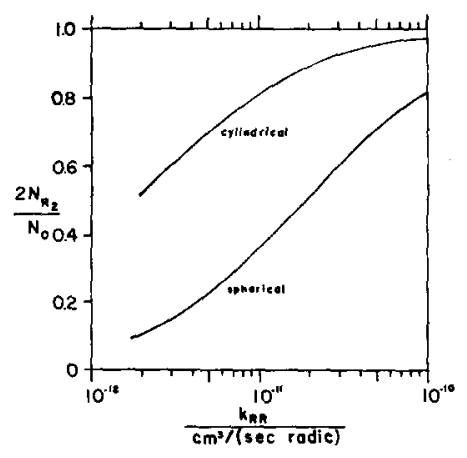

Therefore, when the lack-of-solute-depletion hypothesis is valid (see Sec. 4), the effect on yields of a change in $k_{\mathrm{RR}}$ is equal to the effect of an equal relative change in $N_{0}$. As a result, the curves for $r_{0}=10 \mathrm{~A}$ in Figs. 20 and 21 can be considered to represent the variation of $2 N_{\mathrm{R}_{2}} / N_{0}$ with $k_{\mathrm{RR}}$, after the units of the horizontal axis are adequately changed.

Finally, the variation of yields with $k_{\mathrm{Rs}}$ should be considered. The set of chemical parameters $k_{\mathrm{RS}}, k_{\mathrm{RR}}$, $D_{\mathrm{R}}, D_{\mathrm{S}}, N_{0}, r_{0}, c_{\mathrm{s}_{0}}$ will furnish the same yields as the alternate set $k_{\mathrm{RS}}, k_{\mathrm{RR}^{\prime}}, D_{\mathrm{R}}, D_{\mathrm{B}}, N_{0}{ }^{\prime}, r_{0}, c_{\mathrm{S}_{0}}{ }^{\prime} . N_{0}{ }^{\prime}$ and $c_{\mathrm{S}_{0}}{ }^{\prime}$ are obtained by multiplying the corresponding unprimed quantities by $k_{\mathrm{Rs}}{ }^{\prime} / k_{\mathrm{Rs}}$, and $k_{\mathrm{RR}^{\prime}}$ is obtained by dividing $k_{\mathrm{RR}}$ by this ratio. When the lack-of-solutedepletion hypothesis is valid, an increase of $k_{\mathrm{RS}}$ is obviously equivalent to an equal decrease in $c_{\mathbf{S}_{0}}$. For this situation, the dashed curves of Fig. 13 also represent the variation of $2 N_{\mathrm{R}_{2}} / N_{0}$ with $1 / k_{\mathrm{RS}}$, once the units on the horizontal axis are correctly changed.

\section{CONCLUDING REMARKS}

In this paper we have examined the effect of parameter variations on the results of diffusion-kinetic calculations for a one-radical-one-solute model of the radiation chemistry of dilute aqueous solutions. It is hoped that in this way a feeling for the quantitative features of the model has been developed. A comparison between this model and experiment will be given in a subsequent paper.

\section{ACKNOWLEDGMENTS}

The authors wish to thank Gene P. Reck, F. Wise, and C. H. Gregg, Jr., for help in the preparation of the figures of this paper. 